\title{
Bulk Lorentz factors of gamma-ray bursts
}

\author{
G. Ghirlanda ${ }^{1,2}$, F. Nappo ${ }^{3,1}$, G. Ghisellini ${ }^{1}$, A. Melandri ${ }^{1}$, G. Marcarini ${ }^{2}$, \\ L. Nava ${ }^{1,5}$, O. S. Salafia ${ }^{2,1}$, S. Campana ${ }^{1}$, and R. Salvaterra ${ }^{4,5}$ \\ 2 Dipartimento di Fisica G. Occhialini, Università di Milano Bicocca, Piazza della Scienza 3, 20126 Milano, Italy
}

1 INAF-Osservatorio Astronomico di Brera, via E. Bianchi 46, 23807 Merate, Italy e-mail: giancarlo.ghirlanda@brera.inaf.it

3 Università degli Studi dell'Insubria, via Valleggio 11, 22100 Como, Italy

4 INAF-IASF Milano, via E. Bassini 15, 20133 Milano, Italy

5 INAF-Osservatorio Astronomico di Trieste, via G.B. Tiepolo, 11, 34143 Trieste, Italy

Received 19 July 2017 / Accepted 11 October 2017

\begin{abstract}
Knowledge of the bulk Lorentz factor $\Gamma_{0}$ of gamma-ray bursts (GRBs) allows us to compute their comoving frame properties shedding light on their physics. Upon collisions with the circumburst matter, the fireball of a GRB starts to decelerate, producing a peak or a break (depending on the circumburst density profile) in the light curve of the afterglow. Considering all bursts with known redshift and with an early coverage of their emission, we find 67 GRBs (including one short event) with a peak in their optical or GeV light curves at a time $t_{\mathrm{p}}$. For another $106 \mathrm{GRBs}$ we set an upper limit $t_{\mathrm{p}}^{\mathrm{UL}}$. The measure of $t_{\mathrm{p}}$ provides the bulk Lorentz factor $\Gamma_{0}$ of the fireball before deceleration. We show that $t_{\mathrm{p}}$ is due to the dynamics of the fireball deceleration and not to the passage of a characteristic frequency of the synchrotron spectrum across the optical band. Considering the $t_{\mathrm{p}}$ of 66 long GRBs and the 85 most constraining upper limits, we estimate $\Gamma_{0}$ or a lower limit $\Gamma_{0}^{\mathrm{LL}}$. Using censored data analysis methods, we reconstruct the most likely distribution of $t_{\mathrm{p}}$. All $t_{\mathrm{p}}$ are larger than the time $T_{\mathrm{p}, \gamma}$ when the prompt $\gamma$-ray emission peaks, and are much larger than the time $T_{\mathrm{ph}}$ when the fireball becomes transparent, that is, $t_{\mathrm{p}}>T_{\mathrm{p}, \gamma}>T_{\mathrm{ph}}$. The reconstructed distribution of $\Gamma_{0}$ has median value $\sim 300$ (150) for a uniform (wind) circumburst density profile. In the comoving frame, long GRBs have typical isotropic energy, luminosity, and peak energy $\left\langle E_{\text {iso }}\right\rangle=3(8) \times 10^{50} \mathrm{erg},\left\langle L_{\text {iso }}\right\rangle=3(15) \times 10^{47} \mathrm{erg} \mathrm{s}^{-1}$, and $\left\langle E_{\text {peak }}\right\rangle=1(2) \mathrm{keV}$ in the homogeneous (wind) case. We confirm that the significant correlations between $\Gamma_{0}$ and the rest frame isotropic energy $\left(E_{\text {iso }}\right)$, luminosity $\left(L_{\text {iso }}\right)$, and peak energy $\left(E_{\mathrm{p}}\right)$ are not due to selection effects. When combined, they lead to the observed $E_{\mathrm{p}}-E_{\text {iso }}$ and $E_{\mathrm{p}}-L_{\text {iso }}$ correlations. Finally, assuming a typical opening angle of 5 degrees, we derive the distribution of the jet baryon loading which is centered around a few $10^{-6} M_{\odot}$.
\end{abstract}

Key words. gamma-ray burst: general - radiation mechanisms: non-thermal - relativistic processes

\section{Introduction}

The relativistic nature of gamma-ray bursts (GRBs) was originally posed on theoretical grounds (Goodman 1986; Paczynski 1986; Krolik \& Pier 1991; Fenimore et al. 1993; Baring \& Harding 1997; Lithwick \& Sari 2001): the small size of the emitting region, as implied by the observed millisecond variability, would make the source opaque due to $\gamma-\gamma$ pair production unless it expands with bulk Lorentz factor $\Gamma_{0} \sim$ 100-1000 (e.g. Piran 1999). Refinements of this argument applied to specific GRBs (Abdo et al. 2009a,b; Ackermann et al. 2010a; Hascoët et al. 2012; Zhao et al. 2011; Zou \& Piran 2010; Zou et al. 2011; Tang et al. 2015) led to some estimates of $\Gamma_{0}$. A direct confirmation that GRB outflows are relativistic was found in 970508 (Frail et al. 1997): the suppression of the observed radio variability ascribed to scintillation induced by Galactic dust provided an estimate of the source relativistic expansion. Similarly, the long-term monitoring in the radio band of GRB 030329 (Pihlström et al. 2007; Taylor et al. 2004, 2005) allowed to set limits on the expansion rate (Mesler et al. 2012).

In the standard fireball scenario, after an optically thick acceleration phase the ejecta coast with constant bulk Lorentz factor $\Gamma_{0}$ before decelerating due to the interaction with the external medium. $\Gamma_{0}$ represents the maximum value attained by the outflow during this dynamical evolution (see Kumar \& Zhang for a recent review). Direct estimates of $\Gamma_{0}$ became possible in the last decade thanks to the early follow up of the afterglow emission. The detection of an early afterglow peak, $t_{\mathrm{p}} \sim 150-200 \mathrm{~s}$, in the near-infrared light curve of GRB 060418 and GRB 060607A provided one of the first estimates of $\Gamma_{0}$ (Molinari et al. 2007).

The recent development of networks of robotic telescopes (ROTSE-III: Akerlof et al. 2003; GROCSE: Park et al. 1997; TAROT: Klotz et al. 2009; SkyNet: Graff et al. 2014; WIDGET: Urata et al. 2011; MASTER: Lipunov et al. 2004; Pi of the Sky: Burd et al. 2005; RAPTOR: Vestrand et al. 2002; REM: Zerbi et al. 2001; Watcher: Ferrero et al. 2010) has allowed us to follow up the early optical emission of GRBs. Systematic studies (Liang et al. 2010; Lü et al. 2012; Ghirlanda et al. 2012, G12 hereafter) derived the distribution of $\Gamma_{0}$ and its possible correlation with other observables. G12 found:

1. that different distributions of $\Gamma_{0}$ are obtained according to the density profile of the circumburst medium;

2. the existence of a correlation $\Gamma_{0}^{2} \propto L_{\text {iso }}$ (tighter with respect to that with $E_{\text {iso }}$ );

3. the presence of a linear correlation $\Gamma_{0} \propto E_{\mathrm{p}}$.

As proposed by G12, the combination of these correlations provides a possible interpretation of the spectral energy correlations $E_{\mathrm{p}}-E_{\text {iso }}$ (Amati et al. 2002) and $E_{\mathrm{p}}-L_{\text {iso }}$ (Yonetoku et al. 2004) as the result of larger $\Gamma_{0}$ in bursts with larger luminosity/energy 
and peak energy. Possible interpretations of the correlations between $\Gamma_{0}$ and the GRB luminosity have been proposed in the context of neutrino or magneto-rotation powered jets (Lü et al. 2012; Lei et al. 2013). G12 (see also Ghirlanda et al. 2013a) showed that a possible relation between $\Gamma_{0}$ and the jet opening angle $\theta_{\text {jet }}$ could also justify the $E_{\mathrm{p}}-E_{\gamma}$ correlation (here $E_{\gamma}$ is the collimation corrected energy).

In order to estimate $\Gamma_{0}$, we need to measure the onset time $t_{\mathrm{p}}$ of the afterglow. If the circumburst medium is homogeneous, this is revealed by an early peak in the light curve, corresponding to the passage from the coasting to the deceleration phase of the fireball. On the other hand, if a density gradient due to the progenitor wind is present, the bolometric light curve is constant until the onset time. However, also in the wind case, a peak could be observed if pair production ahead of the fireball is a relevant effect as discussed in G12, for example.

G12 considered 28 GRBs with a clear peak in their optical light curve, and included three GRBs with a peak in their GeV light curves (as observed by the Large Area Telescope - LAT - on board Fermi). Early $t_{\mathrm{p}}$ measurements are limited by the time needed to start the follow-up observations. The LAT $(0.1-$ $100 \mathrm{GeV}$ ), with its large field of view, performs observations simultaneously to the GRB prompt emission for GRBs happening within its field of view. The detection of an early peak in the $\mathrm{GeV}$ light curve, if interpreted as afterglow from the forward shock (e.g. Ghisellini et al. 2010; Kumar \& Barniol Duran 2010), provides the estimate of the earliest $t_{\mathrm{p}}$ (i.e. corresponding to the largest $\Gamma_{0}$ ). In the short GRB 090510, the LAT light curve peaks at $\sim 0.2 \mathrm{~s}$ corresponding to $\Gamma_{0} \sim 2000$ (Ghirlanda et al. 2010; Ackermann et al. 2010b). Recently it has been shown that upper limits on $\Gamma_{0}$ can be derived from the non-detection of GRBs by the LAT (Nava et al. 2017) and that such limits are consistent with lower limits and detections reported in the literature.

Precise and fast localisations of GRB counterparts, routinely performed by Swift, coupled to efficient follow up by robotic telescope networks, allowed us to follow the optical emission starting relatively soon after the GRB trigger. However, a delay of a few hundred seconds can also induce a bias against the measure of early-intermediate $t_{\mathrm{p}}$ values (Hascoët et al. 2012). As argued by Hascoët et al. (2012), the distribution of $\Gamma_{0}$, derived through measured $t_{\mathrm{p}}$, could lack intermediate to large values of $\Gamma_{0}$ (corresponding to intermediate to early values of $t_{\mathrm{p}}$ ) and the $\Gamma_{0}-E_{\text {iso }}$ correlation could be a boundary, missing several bursts with large $\Gamma_{0}$ (i.e. because of the lack of early $t_{\mathrm{p}}$ measurements).

For this reason, upper limits are essential to derive the distribution of $\Gamma_{0}$ in GRBs and its possible correlation with other prompt emission properties $\left(E_{\text {iso }}, L_{\text {iso }}\right.$, and $\left.E_{\mathrm{p}}\right)$ and, in general, to study the comoving frame properties of the population. To this aim, in this paper (i) we collect the available bursts with an optical $t_{\mathrm{p}}$, expanding and revising the previously published samples; and (ii) we collect a sample of bursts with upper limits on $t_{\mathrm{p}}$. Through this censored data sample we reconstruct the distribution of $\Gamma_{0}$ accounting (for the first time) for upper limits. We then employ Monte Carlo methods to study the correlations between $\Gamma_{0}$ and the rest frame isotropic energy/luminosity and peak energy.

The sample selection and its properties are presented in Sects. 2-4, respectively. The different formulae for the estimate of the bulk Lorentz factor $\Gamma_{0}$ appearing in the literature are presented and compared in Sect. 5. In Sect. 6 the distribution of $\Gamma_{0}$ and its correlation (Sect. 7) with $E_{\mathrm{iso}}, L_{\mathrm{iso}}$, and $E_{\mathrm{p}}$ are studied. Discussion and conclusions follow in Sect. 8. We assume a flat cosmology with $h=\Omega_{\Lambda}=0.7$.

\section{The sample}

We consider GRBs with measured redshift $z$ and well constrained spectral parameters of the prompt emission. For these events it is possible to estimate the isotropic energy $E_{\text {iso }}$ and luminosity $L_{\text {iso }}$ and the rest frame peak spectral energy $E_{\mathrm{p}}$ (i.e. the peak of the $v F_{v}$ spectrum).

$\Gamma_{0}$ can be estimated from the measure of the peak $t_{\mathrm{p}}$ of the afterglow light curve interpreted as due to the deceleration of the fireball. We found in the literature 67 GRBs (66 long and 1 short) with an estimate of $t_{\mathrm{p}}$ (see Table A.1). Of these, $59 t_{\mathrm{p}}$ are obtained from the optical and 8 from the GeV light curves. Through a systematic search of the literature we collected 106 long GRBs whose optical light curve, within one day of the trigger, decays with no apparent $t_{\mathrm{p}}$. These GRBs provide upper limits $t_{\mathrm{p}}^{\mathrm{UL}}$. Details of the sample selection are reported in the following sections.

\subsection{Afterglow onset $t_{p}$}

G12 studied a sample of 30 long GRBs, with $t_{\mathrm{p}}$ measured from the optical (27 events) or from the $\mathrm{GeV}$ (3 events) light curves. We revise the sample of G12 with new data recently appearing in the literature, and we extend it, beyond GRB 110213A, including all new GRBs up to July 2016 with an optical or GeV afterglow light curve showing a peak $t_{\mathrm{p}}$.

Bursts with a peak in their early X-ray emission are not included in our final sample because the X-ray can be dominated (a) by an emission component of "internal" origin, for example, due to the long lasting central engine activity (e.g. Ghisellini et al. 2007; Genet et al. 2007; Ioka et al. 2006; Panaitescu 2008; Toma et al. 2006; Nardini et al. 2010) and/or (b) by bright flares (Margutti et al. 2010) ${ }^{1}$.

We excluded from our final sample (i) bursts with a multipeaked optical light curve at early times ${ }^{2}$; and (ii) events with an optical peak preceded by a decaying light curve (e.g. GRB 100621A, GRB 080319C present in G12) since the early decay suggests the possible presence of a multi-peaked structure. The latter events, however, were included in the sample of $t_{\mathrm{p}}^{\mathrm{UL}}$ (Sect. 2.2), considering the earliest epoch of their optical decay.

\subsubsection{The gold sample}

Table A.1 lists all the GRBs we collected. The "Gold" sample is composed of sources with a complete set of information, namely measured $t_{\mathrm{p}}(\mathrm{Col} .6)$ and spectral parameters (Cols. 3-5). It contains 49 events: 48 long GRBs plus the short event 090510. GRBs of the Gold sample have the label " $(\mathrm{g})$ " at the end of their names reported in Col. 1 of Table A.1. The redshift $z$, rest frame peak energy $E_{\mathrm{p}}$, isotropic energy and luminosity ( $E_{\text {iso }}$ and $L_{\text {iso }}$, respectively) are given in Table A.1. Eight out of 49 GRBs have their $t_{\mathrm{p}}$ measured from the GeV light curve as observed by the Fermi-LAT (labelled "L" or "SL" for the short GRB 090510).

For GRB 990123, GRB 080319B, and GRB 090102 reported in Table A.1, it has been proposed that the early optical emission (and the observed peak) is produced by either the reverse shock (RS; Bloom et al. 2009; Japelj et al. 2014; Sari \& Piran 1999) or

\footnotetext{
1 Liang et al. (2010), Lü et al. (2012) and Wu et al. (2011) include in their samples also bursts with a peak in the X-ray light curve thus resulting in a larger but less homogeneous and secure sample of $t_{\mathrm{p}}$. 2 In these events $\Gamma_{0}$ might still be estimated, but only under some assumptions on the dynamical evolution of the burst outflow (e.g. GRB 090124 - Nappo et al. 2014).
} 
by a combination of forward and reverse shock (Gendre et al. 2010; Steele et al. 2009) or even by a two-component jet structure (e.g. Racusin et al. 2008, for GRB 080319B). We assume for these three GRBs that the peak is due to the outflow deceleration and include them in our sample.

\subsubsection{The silver sample}

In our search we found 18 events with $t_{\mathrm{p}}$ but with poorly constrained prompt emission properties $\left(E_{\mathrm{p}}, E_{\mathrm{iso}}\right.$, and $\left.L_{\text {iso }}\right)$. In most of these cases, the Swift Burst Alert Telescope (BAT) limited (15-150 keV) energy band coupled with a relatively low flux of the source prevent us from constraining the peak of the spectrum $\left(E_{\mathrm{p}}\right)$ even when it lies within the BAT energy range. Most of these BAT spectra were fitted by a simple power law model. Sakamoto et al. (2011) showed that, also in these cases, the $E_{\mathrm{p}}$ could be derived adopting an empirical correlation between the spectral index of the power law, fitted to the BAT spectrum, and $E_{\mathrm{p}}$. This empirical correlation was derived and calibrated with those bursts where BAT can measure $E_{\mathrm{p}}$. Alternatively, Butler et al. (2007, 2010) proposed a Bayesian method to recover the value of $E_{\mathrm{p}}$ for BAT spectra fitted by the simple power law model.

We adopted the values of $E_{\mathrm{p}}$ and $E_{\text {iso }}$ calculated by Butler et al. $(2007,2010)$ for $15 / 18$ GRBs in common with their list and the Sakamoto et al. (2011) relation for the remaining $3 / 18$ events in order to exploit the measure of $t_{\mathrm{p}}$ also for these 18 bursts. Sakamoto et al. (2011) and Butler et al. (2007, 2010) study the time-integrated spectrum of GRBs. We estimated the luminosity $L_{\text {iso }}=E_{\text {iso }}(P / F)$, where $P$ and $F$ are the peak flux and fluence, respectively, in the $15-150 \mathrm{keV}$ energy range. GRBs of the "Silver" sample are labelled "(s)" in Table A.1.

While we made this distinction explicit for clarity, in what follows we use the total sample of $t_{\mathrm{p}}$ without any further distinction between the Gold and Silver samples.

\subsection{Upper limits on $t_{p}$}

The afterglow onset is expected within one day for typical GRB parameters (see Sect. 4). Several different observational factors, however, can prevent the measure of $t_{\mathrm{p}}$. It is hard to construct a sample of upper limits $t_{\mathrm{p}}^{\mathrm{UL}}$. Hascoët et al. 2014 included some $t_{\mathrm{p}}$ in their analysis but without a systematic selection criterion.

In this paper we collect from the literature all the GRBs with known $z$ and with an optical counterpart observed at least three times within one day of the trigger. If the light curve is decaying in time we set the upper limit $t_{\mathrm{p}}^{\mathrm{UL}}$ corresponding to the earliest optical observation. Similar criteria apply if the long-lived afterglow emission is detected in the GeV energy range by the LAT. For several recent bursts, highly sampled early light curves are available. We excluded events with complex optical emission at early times and selected only those with an indication of a decaying optical flux.

The 106 GRBs with $t_{\mathrm{p}}^{\mathrm{UL}}$ are reported in Table A.1. For the purposes of our analysis in the following we use a subsample of the 85 most constraining $t_{\mathrm{p}}^{\mathrm{UL}}$, that is, those with $t_{\mathrm{p}}^{\mathrm{UL}} \leq 11500 \mathrm{~s}$ which corresponds to five times the largest value of $t_{\mathrm{p}}$ of the Gold+Silver sample.

\section{Sample properties}

In this section we present the distribution of $t_{\mathrm{p}}$ (in the observer and rest frame) and study the possible correlation of the rest frame $t_{\mathrm{p}}$ with the observables of the prompt emission.

\subsection{Distribution of the observer frame $t_{p}$}

Figure 1 shows the cumulative distribution (red line) of the observer frame afterglow peak time $t_{\mathrm{p}}$ of long GRBs ${ }^{3}$. The distribution of upper limits $t_{\mathrm{p}}^{\mathrm{UL}}$ is shown by the dashed black line (with leftward arrows). The distribution of measured $t_{\mathrm{p}}$ is consistent with that of the upper limits at the extremes, that is, below $30 \mathrm{~s}$ and above $\sim 1000 \mathrm{~s}$. In particular, the low-end of the distribution of $t_{\mathrm{p}}$ is mainly composed of bursts whose onset time is provided by the LAT data. Considering only GRBs with measured $t_{\mathrm{p}}$ (red line in Fig. 1), the (log) average $t_{\mathrm{p}} \sim 230 \mathrm{~s}$ while upper limits (dashed black line in Fig. 1) have a (log) average $t_{\mathrm{p}} \sim 160 \mathrm{~s}$. The relative position of the two distributions (red and black dashed) suggests that if we considered only $t_{\mathrm{p}}$ measurements (as in G12; Liang et al. 2010; Lü et al. 2012) we would miss several intermediate-early onsets. This is confirmed also if we consider only the GRBs present in our sample which are part of the so called "BAT6" sample (Salvaterra et al. 2012). Indeed, this high flux cut sample of 58 Swift GRBs is $90 \%$ complete in redshift. There are $16 \mathrm{GRBs}$ in our sample with measured $t_{\mathrm{p}}$ and 34 with $t_{\mathrm{p}}^{\mathrm{UL}}$ in common with the BAT6 sample (i.e. $86 \%$ of the sample). Their $t_{\mathrm{p}}$ distribution (and the distribution of their $t_{\mathrm{p}}^{\mathrm{UL}}$ ) is shown in the insert of Fig. 1. Similarly to the larger sample, the distribution of $t_{\mathrm{p}}$ for the BAT6 sample is close to that of upper limits $t_{\mathrm{p}}^{\mathrm{UL}}$.

In our sample nearly half of the bursts have $t_{\mathrm{p}}$ measured and half are upper limits. The distributions of $t_{\mathrm{p}}$ and $t_{\mathrm{p}}^{\mathrm{UL}}$ overlap considerably ensuring that random censoring is present. Survival analysis (Feigelson \& Nelson 1985) can be used to reconstruct the true distribution of $t_{\mathrm{p}}$. We use the non-parametric KaplanMeier estimator (KM), as adapted by Feigelson \& Nelson (1985) to deal with upper limits. The KM reconstructed CDF is shown by the solid black line in Fig. 1 . The $95 \%$ confidence interval on this distribution (Miller 1981; Kalbfleish \& Prentice 1980) is shown by the yellow shaded region in Fig. 1. The median value of the $\mathrm{CDF}$ is $\left\langle t_{\mathrm{p}}\right\rangle=60 \pm 20 \mathrm{~s}$ ( $1 \sigma$ uncertainty). We verified that, considering a more stringent subsample of upper limits, that is, $t_{\mathrm{p}}^{\mathrm{UL}} \leq 2 \times \max \left(t_{\mathrm{p}}\right)$, similar CDF and average values are obtained.

\subsection{Distribution of the rest frame $t_{p}$}

Figure 2 shows the cumulative distribution of $t_{\mathrm{p}}$ in the rest frame. Colour and symbols are the same as in Fig. 1. In particular we note that also in the rest frame the cumulative distribution of measured $t_{\mathrm{p}}$ (solid red line) is close to the distribution of upper limits (leftward arrows). The KM estimator leads to a reconstructed rest frame $t_{\mathrm{p}}$ distribution (solid black-yellow shaded curve) which is distributed between 1 and $10^{3} \mathrm{~s}$ with an average value of $20_{-5}^{+10} \mathrm{~s}$. The insert of Fig. 2 shows the distribution of the onset time of the GRBs belonging to the complete Swift sample. Again the measured $t_{\mathrm{p}}$ distribution (solid blue line) is close to the

3 The short GRB 090510 is not included in the distributions. Its onset time $t_{\mathrm{p}}=0.2 \mathrm{~s}$ Ghirlanda et al. (2010) would place it in the lowest bin of the distribution. 


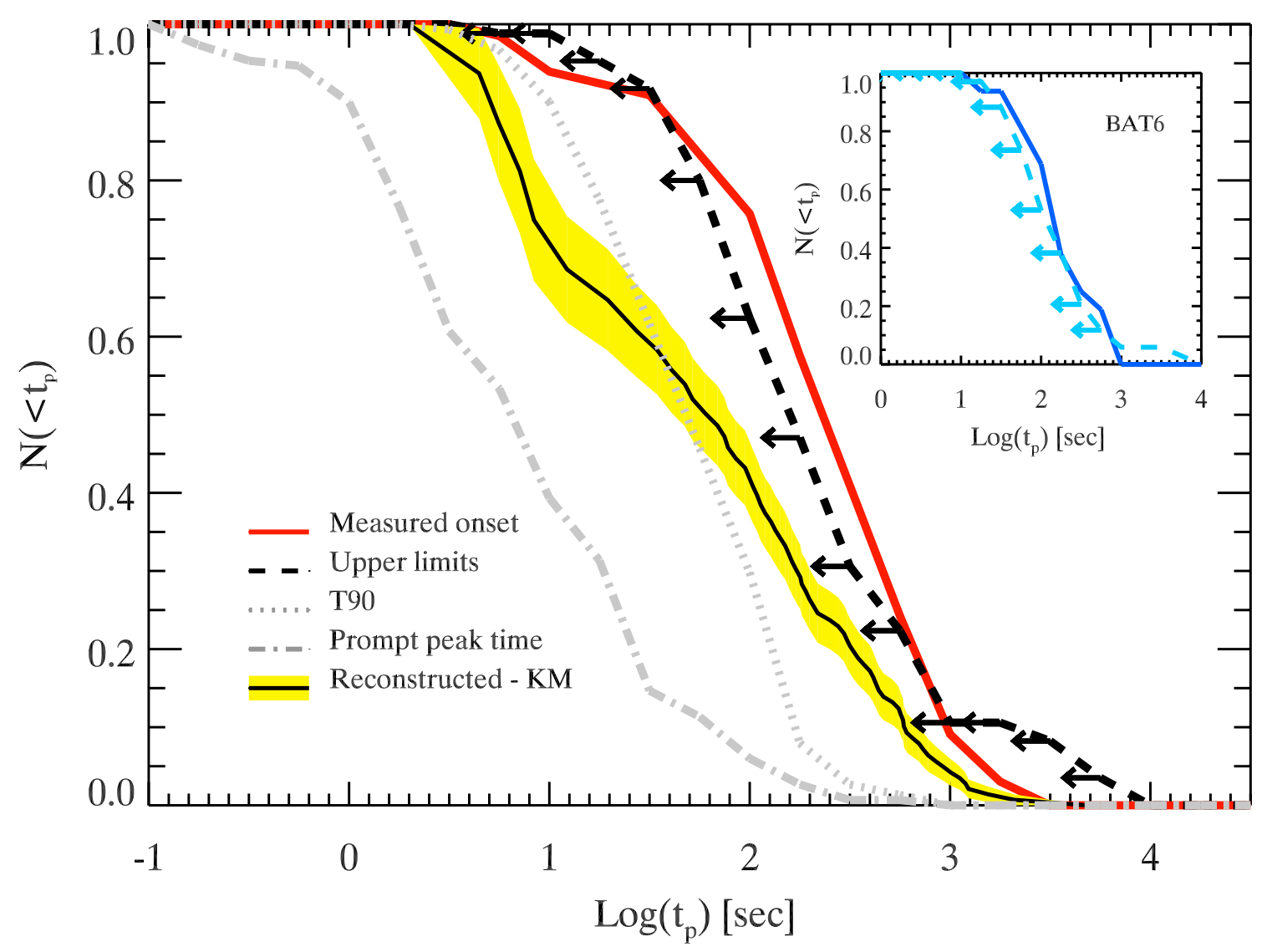

Fig. 1. Cumulative distribution of the afterglow onset time $t_{\mathrm{p}}$ (red solid line) in the observer frame for the 66 long GRBs of the "Gold+Silver" sample. The black dashed line (marked with leftward arrows) is the cumulative distribution of 85 upper limits on $t_{\mathrm{p}}$ (filtered from Table A.1 according to $t_{\mathrm{p}}^{\mathrm{UL}} \leq 5 \times \max \left(t_{\mathrm{p}}\right) \sim 11500 \mathrm{~s}$ ). The black solid line (shaded yellow region) is the reconstructed $t_{\mathrm{p}}$ distribution (95\% confidence interval) accounting for $t_{\mathrm{p}}^{\mathrm{UL}}$ through the Kaplan Meier estimator. The dotted and dot-dashed lines are the cumulative distributions of $T_{90}$ and $T_{\mathrm{p}, \gamma}$ (i.e. the time of the peak of the prompt emission light curve), respectively, for the 151 long GRBs. The distributions have been normalised to the respective sample size. Insert: Distribution of $t_{\mathrm{p}}$ (solid blue line) and of upper limits $t_{\mathrm{p}}^{\mathrm{UL}}$ (dashed cyan line with leftward arrows) of the $50 \mathrm{GRBs}$ of our sample (16 with $t_{\mathrm{p}}$ and 34 with $t_{\mathrm{p}}^{\mathrm{UL}}$ ) also present in the Swift BAT complete sample (Salvaterra et al. 2012).

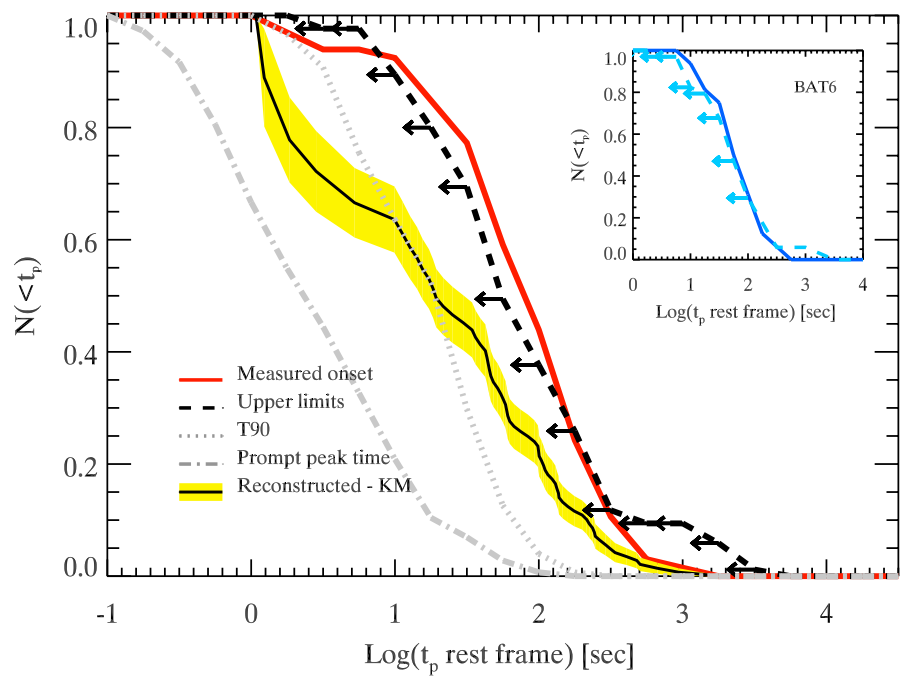

Fig. 2. Cumulative distribution of the afterglow onset time $t_{\mathrm{p}}$ in the rest frame. Same symbols and colour code as in Fig. 1.

distribution of upper limits, suggesting the presence of a selection bias against the measurement of the earliest $t_{\mathrm{p}}$ values which is, however, not due to the requirement of the measure of the redshift.

\subsection{Comparison between $t_{p}, T_{90}$, and $T_{p, \gamma}$}

One assumption for the estimate of $\Gamma_{0}$ from the measure of $t_{\mathrm{p}}$ (see Sect. 4) is that most of the kinetic energy of the ejecta has been transferred to the blast wave (so called "thin shell approximation" - Hascoët et al. 2014) which is decelerated by the circumburst medium. Therefore, we should expect that $t_{\mathrm{p}}$ be larger than the duration of the prompt emission, estimated by $T_{90}$. To check this hypothesis, we collected $T_{90}$ for the bursts of our sample: its distribution is shown by the grey dotted line in Fig. 1. A scatter plot showing $T_{90}$ vs. the observer frame $t_{\mathrm{p}}$ is shown in the top panel of Fig. 3: bursts with measured $t_{\mathrm{p}}$ are shown by the red filled circles (GRBs with $t_{\mathrm{p}}$ derived from the GeV-LAT light curve are shown by the star symbols), upper limits $t_{\mathrm{p}}^{\mathrm{UL}}$ are also shown by the black (green for LAT bursts) symbols. The majority $(80 \%)$ of GRBs lie below the equality line (dashed line in Fig. 3) having $t_{\mathrm{p}}>T_{90} .20 \%$ of the bursts have $t_{\mathrm{p}}<T_{90}$. A generalised Spearman's rank correlation test (accounting also for upper limits - Isobe et al. $(1986,1990))$ indicates no significant correlation between $T_{90}$ and $t_{\mathrm{p}}$ (at $>3 \sigma$ level of confidence).

The prompt emission of GRBs can be highly structured with multiple peaks separated by quiescent times. While $T_{90}$ is representative of the overall duration of the burst, another interesting timescale is the peak time of the prompt emission light curve $T_{\mathrm{p}, \gamma}$. This time corresponds to the emission of a considerable fraction of energy during the prompt and it is worth comparing 
G. Ghirlanda et al.: Bulk Lorentz factor of GRBs

Table 1. Correlation between rest frame $t_{\mathrm{p}} /(1+z)$ and prompt emission properties (Fig. 4).

\begin{tabular}{lcccc|cccc}
\hline \hline Correlation & \multicolumn{3}{c}{$t_{\mathrm{p}}$ only } & \multicolumn{3}{c}{$t_{\mathrm{p} \& t_{\mathrm{p}}^{\mathrm{UL}}}$} \\
& $r$ & $P$ & $m$ & $q$ & $r$ & $P$ & $m$ & $q$ \\
\hline$t_{\mathrm{p}} /(1+z)$ vs. $E_{\text {iso }}$ & -0.54 & $3 \times 10^{-6}$ & $-0.71 \pm 0.06$ & $39.70 \pm 3.28$ & -0.20 & $1 \times 10^{-2}$ & $-0.95 \pm 0.01$ & $51.61 \pm 1.00$ \\
$t_{\mathrm{p}} /(1+z)$ vs. $L_{\text {iso }}$ & -0.62 & $1 \times 10^{-7}$ & $-0.67 \pm 0.10$ & $36.89 \pm 5.10$ & -0.44 & $2 \times 10^{-8}$ & $-0.87 \pm 0.02$ & $46.77 \pm 1.00$ \\
$t_{\mathrm{p}} /(1+z)$ vs. $E_{\mathrm{p}}$ & -0.54 & $3 \times 10^{-6}$ & $-1.25 \pm 0.12$ & $5.11 \pm 0.33$ & -0.24 & $3 \times 10^{-3}$ & $-1.25 \pm 0.03$ & $4.70 \pm 0.10$ \\
\hline
\end{tabular}

Notes. The Spearman's correlation correlation coefficient $r$ and its chance probability $P$ are reported considering only 66 long GRBs with estimated $t_{\mathrm{p}}$ or including also the 85 upper limits $t_{\mathrm{p}}^{\mathrm{UL}}$. The correlation slope $m$ and intercept $q$ of the model $Y=q+m X$ with their $1 \sigma$ uncertainties are reported.

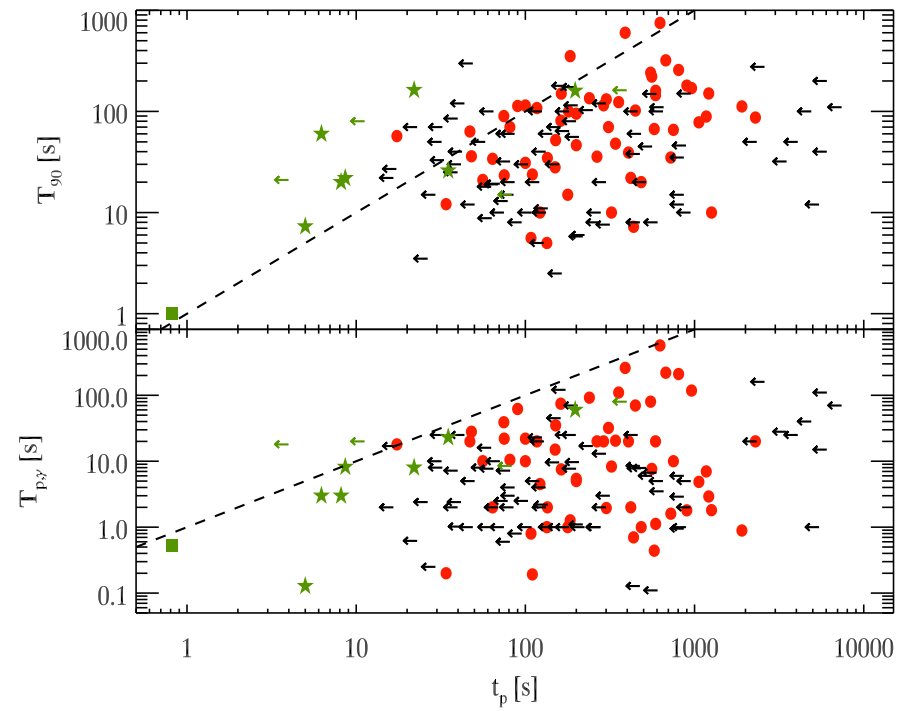

Fig. 3. Top panel: GRB duration $T_{90}$ vs. afterglow peak time $t_{\mathrm{p}}$. GRBs with measured $t_{\mathrm{p}}$ are shown with red circles (green stars for LAT bursts). Upper limits on $t_{\mathrm{p}}$ are shown by the black arrows (green arrows for LAT GRBs). Bottom panel: time of the peak of the prompt emission light curve $T_{\mathrm{p}, \gamma}$ vs. $t_{\mathrm{p}}$. Same symbols and colours as in the top panel. In both panels the equality is shown by the dashed line and the short GRB 090510 is shown by the green square.

it with $t_{\mathrm{p}}$. The distribution of $T_{\mathrm{p}, \gamma}$ is shown by the dot-dashed line in Fig. 1. The bottom panel of Fig. 3 compares $T_{\mathrm{p}, \gamma}$ with $t_{\mathrm{p}}$. Noteworthily, no GRB has $t_{\mathrm{p}}<T_{\mathrm{p}, \gamma}$. There are only two upper limits $t_{\mathrm{p}}^{\mathrm{UL}}$ from the early follow up of the GeV light curve (green arrows in the bottom panel of Fig. 3) which lie above the equality line. However, the large uncertainties in these two bursts (090328 and 091003) on their GeV light curve at early times (Panaitescu 2017) make them also compatible with having $t_{\mathrm{p}}>T_{\mathrm{p}, \gamma}$. Again, the Spearman's generalised test results in no significant correlation between $t_{\mathrm{p}}$ and $T_{\mathrm{p}, \gamma}$.

\subsection{Empirical correlations}

We study the correlation between the onset time $t_{\mathrm{p}}$ in the rest frame and the energetic of GRBs. Figure 4 shows $t_{\mathrm{p}} /(1+z)$ vs. the prompt emission isotropic energy $E_{\mathrm{iso}}$, isotropic luminosity $L_{\text {iso }}$ and rest frame peak energy $E_{\mathrm{p}}$.

GRBs with measured $t_{\mathrm{p}}$ (red circles and green stars in Fig. 4) show significant correlations (chance probabilities $<10^{-5}$ - Table 1) shown by the red solid lines (obtained by a least square fit with the bisector method) in the panels of Fig. 4. The correlation parameters (slope and normalisation) obtained only with $t_{\mathrm{p}}$ are reported in the left part of Table 1.
Upper limits $t_{\mathrm{p}}^{\mathrm{UL}}$ (black downward arrows in Fig. 4) are distributed in the same region of the planes occupied by $t_{\mathrm{p}}$. Figure 4 shows also the lower limits on $t_{\mathrm{p}}^{\mathrm{LL}}$ (grey upward arrows) derived assuming that $t_{\mathrm{p}}>T_{\mathrm{p}, \gamma}$.

The 85 GRBs without a measured $t_{\mathrm{p}}$ should have their onset $T_{\mathrm{p}, \gamma}<t_{\mathrm{p}}<t_{\mathrm{p}}^{\mathrm{UL}}$ corresponding to the vertical interval limited by the grey and black arrows in Fig. 4. Indeed, the reconstructed distribution of $t_{\mathrm{p}}$ (shown by the solid black line in Fig. 1) is bracketed by the cumulative distribution of $T_{\mathrm{p}, \gamma}$ on the left-hand side (dot-dashed grey line in Fig. 1) and by the distribution of $t_{\mathrm{p}}^{\mathrm{UL}}$ on the right-hand side (dashed black line in Fig. 1).

In order to evaluate the correlations of Fig. 4 combining measured $t_{\mathrm{p}}$ and upper/lower limits we adopted a Monte Carlo approach. We assume that the KM estimator provides the distribution of $t_{\mathrm{p}}$ of the population of GRBs shown by the solid black line in Fig. 1. For each of the 85 GRBs with upper limits we extract randomly from the reconstructed $t_{\mathrm{p}}$ distribution a value of $t_{\mathrm{p}}$ (requiring that the extracted value $t_{\mathrm{p}}(i)$ falls within the range $T_{\mathrm{p}, \gamma}(i) \leq t_{\mathrm{p}}(i) \leq t_{\mathrm{p}}^{\mathrm{UL}}(i)$ - where $i$ runs from 1 to 85$)$ and combine them with the $66 \mathrm{GRBs}$ with measured $t_{\mathrm{p}}$ to compute the correlation (using the bisector method). We repeat this random extraction obtaining $10^{5}$ random samples and compute the average values of the Spearman's rank correlation coefficient, of its chance probability, and of the slope and normalisation of the correlations (fitted to the $10^{5}$ randomly generated samples). In Fig. 4, the average correlation obtained through this Monte Carlo method is shown by the dot-dashed black line. The average values of the rank correlation coefficient, its probability, and the correlation parameters (slope and normalisation) are reported in the right section of Table 1 .

These results show that significant correlations exist between the observables (i.e. fully empirical at this stage). A larger energy/luminosity/peak energy corresponds to an earlier $t_{\mathrm{p}}$. The distribution of upper $\left(t_{\mathrm{p}}^{\mathrm{UL}}\right)$ and lower $\left(T_{\mathrm{p}, \gamma}\right)$ limits in the planes of Fig. 4 show that these planes cannot be uniformly filled with points, further supporting the existence of these correlations.

\section{On the origin of the afterglow peak time $t_{\mathrm{p}}$}

In the following we assume that the afterglow peak $t_{\mathrm{p}}$ is produced by the fireball deceleration. However, other effects can produce an early peak in the afterglow light curve: $t_{\mathrm{p}}$ can be due to the passage across the observation band of the characteristic frequencies of the synchrotron spectrum. In this case, however, any of the characteristic synchrotron frequencies should lie very close to the observation band at the time of the peak.

The synchrotron injection frequency is (Panaitescu \& Kumar 2000):

$v_{\text {inj }}(t)=0.92 \times 10^{13} \epsilon_{\mathrm{B},-2}^{1 / 2} \epsilon_{\mathrm{e},-1}^{2}\left(E_{\mathrm{iso}, 53} / \eta\right)^{1 / 2} t^{-3 / 2}(1+z)^{-1} \mathrm{~Hz},(1)$ 


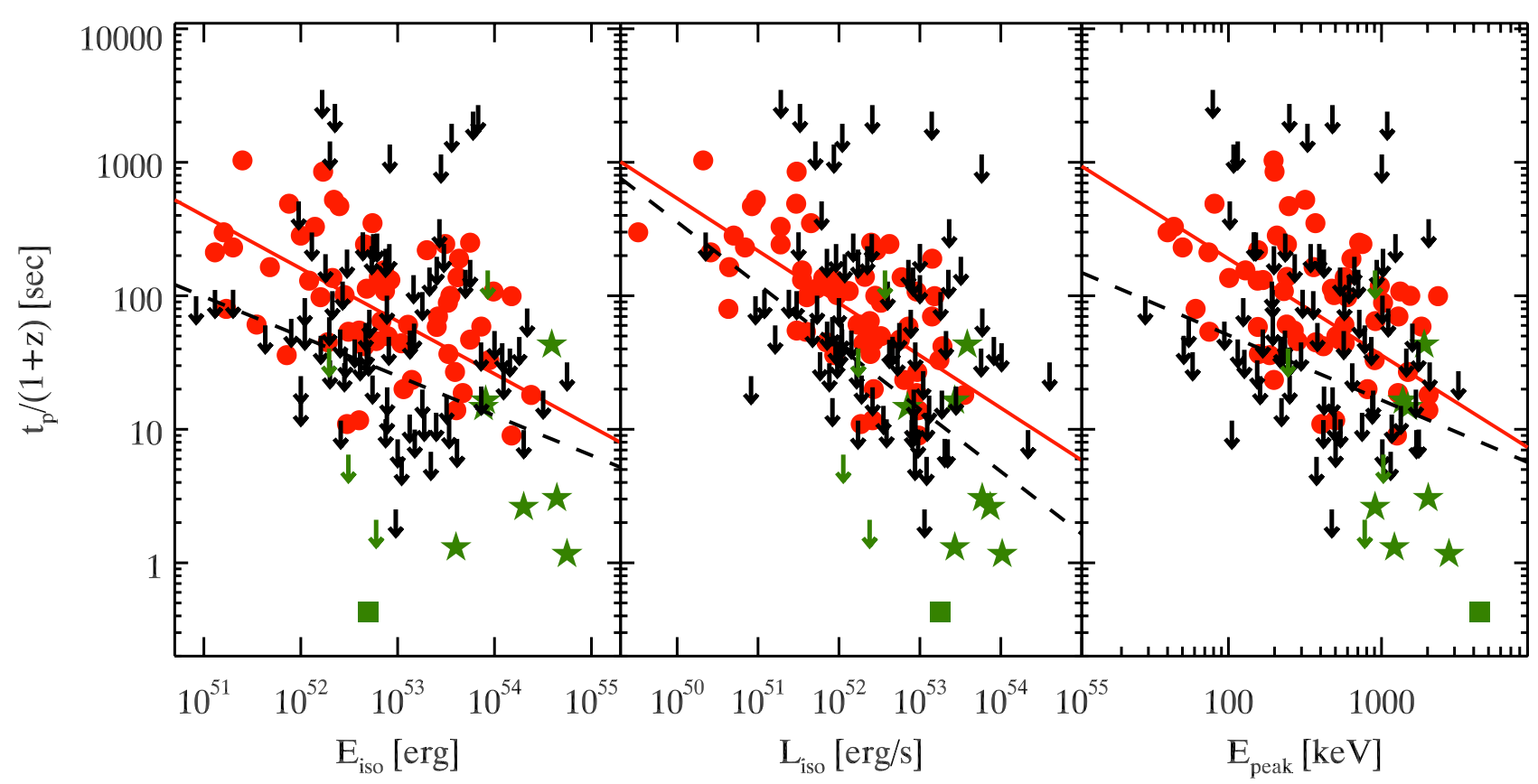

Fig. 4. Left panel: $t_{\mathrm{p}} /(1+z)$ vs. $E_{\mathrm{iso}}$. GRBs with estimates of $t_{\mathrm{p}}$ are shown with filled red circles (green symbols for LAT events). Upper limits on $t_{\mathrm{p}}$ (Table A.1) are shown by the black arrows (green arrows for LAT events). Middle panel: $t_{\mathrm{p}} /(1+z)$ vs. $L_{\mathrm{iso}}$. Right panel: $t_{\mathrm{p}} /(1+z) \mathrm{vs}$. $E_{\mathrm{p}}$. In all panels the short GRB 090510 is shown by the green square symbol.

where $\epsilon_{\mathrm{e}}$ and $\epsilon_{\mathrm{B}}$ represent the fraction of energy shared between electrons and magnetic field at the shock and $\eta$ is the efficiency of conversion of kinetic energy to radiation (i.e. $E_{\text {iso, } 53} / \eta$ represents the kinetic energy in units of $10^{53} \mathrm{ergs}$ in the blast wave). Here $t$ is measured in days in the source rest frame. The above expression for $v_{\text {inj }}(t)$ is valid either if the circumburst medium has constant density or if its density decreases with the distance from the source as $r^{-2}$ (wind medium); in the latter case only the normalisation constant is larger by a factor $\sim 2$.

Figure 5 shows $v_{\text {inj }}\left(t=t_{\mathrm{p}}\right)$ (red and blue symbols for the homogeneous or wind medium case and stars for the LAT bursts) with respect to the optical $R$ frequency (solid horizontal line). We assumed typical values of the shock parameters: $\epsilon_{\mathrm{B}}=0.01$ and $\epsilon_{\mathrm{e}}=0.1$ and an efficiency $\eta=20 \%$ (Nava et al. 2014; Beniamini et al. 2015). The value $\epsilon_{\mathrm{e}}=0.1$ is consistent with Beniamini \& van der Horst (2017) and Nava et al. (2014) who recently found a narrow distribution of this parameter as inferred from the analysis of the radio and $\mathrm{GeV}$ afterglow, respectively. $\epsilon_{\mathrm{B}}$ is less constrained and has a wider dispersion, between $10^{-4}$ and $10^{-1}$ (Granot \& van der Horst 2014; Santana et al. 2014; Zhang et al. 2015; Beniamini et al. 2016), which translates into a factor of 10 for the value of $v_{\text {inj }}$ (vertical lines in Fig. 5) obtained assuming $\epsilon_{\mathrm{B}}=0.01$ (open circles in Fig. 5). In order to account for this uncertainty we show, as vertical lines in Fig. 5 (for the open circles only for clarity), the possible range of frequencies that are obtained assuming $\epsilon_{\mathrm{B}} \in\left[10^{-4}, 10^{-1}\right]$. In all bursts, the injection frequency, when the afterglow peaks (i.e. at $t_{\mathrm{p}}$ ), is above the optical band and it cannot produce the peak as we see $\mathrm{it}^{4}$.

However, one may argue that the above argument depends on the assumed typical values of $\epsilon_{\mathrm{e}}$ and $\epsilon_{\mathrm{B}}$. Consider, for example,

\footnotetext{
4 We note also that for the LAT bursts (star symbols) the injection frequency is a factor 10 below the $\mathrm{GeV}$ band, and also in these cases the peak of the LAT light curve can be interpreted as the deceleration peak (Nava et al. 2017).
}

the homogeneous case $s=0$. In order to "force" $v_{\text {inj }}=v_{\mathrm{opt}, \mathrm{R}}=$ $4.86 \times 10^{14} \mathrm{~Hz}$ at $t=t_{\mathrm{p}}$ and reproduce the observed flux at the peak $F_{\mathrm{p}}$, one would require a density of the ISM:

$n_{0}(s=0)=1.3 \times 10^{3} D_{28}^{4} E_{53}^{-1} \epsilon_{\mathrm{e},-1}^{4} t_{\mathrm{p}, \mathrm{sec},-2}^{-3}(1+z)^{-4 / 3} F_{\mathrm{p}, \mathrm{mJy}}^{2} \mathrm{~cm}^{-3}$,

where $D_{28}$ is the luminosity distance in units of $10^{28} \mathrm{~cm}$ and $t_{\mathrm{p}, \mathrm{sec},-2}$ is the rest frame peak time, now expressed in units of $100 \mathrm{~s}$. For typical peak flux of $10 \mathrm{mJy}$ we should have densities $\sim 10^{5} \mathrm{~cm}^{-3}$.

The other synchrotron characteristic frequency which could evolve and produce a peak when passing across the optical band is the cooling frequency $v_{\text {cool }}$. For the homogeneous medium $v_{\text {cool }}=3.7 \times 10^{14} \epsilon_{\mathrm{B},-2}^{-3 / 2}\left(E_{\mathrm{iso}, 53} / \eta\right)^{-1 / 2} n^{-1} t_{\mathrm{p}}^{-1 / 2}(1+z)^{-1}(Y+1)^{-2}$ $\mathrm{Hz}$, where $n$ is the number density of the circum burst medium and $Y$ the Compton parameter (Panaitescu \& Kumar 2000). In this case, the portion of the synchrotron spectrum which could produce a peak is $v<v_{\text {cool }}$, under the assumption that $v_{\text {cool }}<$ $v_{\text {inj }}$, and the flux evolution should be $F(t) \propto t^{1 / 6}$ (Panaitescu 2017; Sari et al. 1998). This is much shallower than the rising slopes of the afterglow emission of most of the bursts before $t_{\mathrm{p}}$ (Liang et al. 2013). In the wind case, the cooling break increases with time $\propto t^{1 / 2}$ and, if transitioning accross the observing band from below, it should produce a decaying afterglow flux $F(t) \propto t^{-1 / 4}$ and not a peak.

Therefore, we are confident that $t_{\mathrm{p}}$ cannot be produced by any of the synchrotron frequencies crossing the optical band and it can be interpreted as due to the deceleration of the fireball and used to estimate $\Gamma_{0}$.

\section{Estimate of $\boldsymbol{\Gamma}_{0}$}

In this section we revise, in chronological order, the different methods and formulae proposed for estimating the bulk Lorentz factor $\Gamma_{0}$ (Ghirlanda et al. 2012; Ghisellini et al. 2010; 


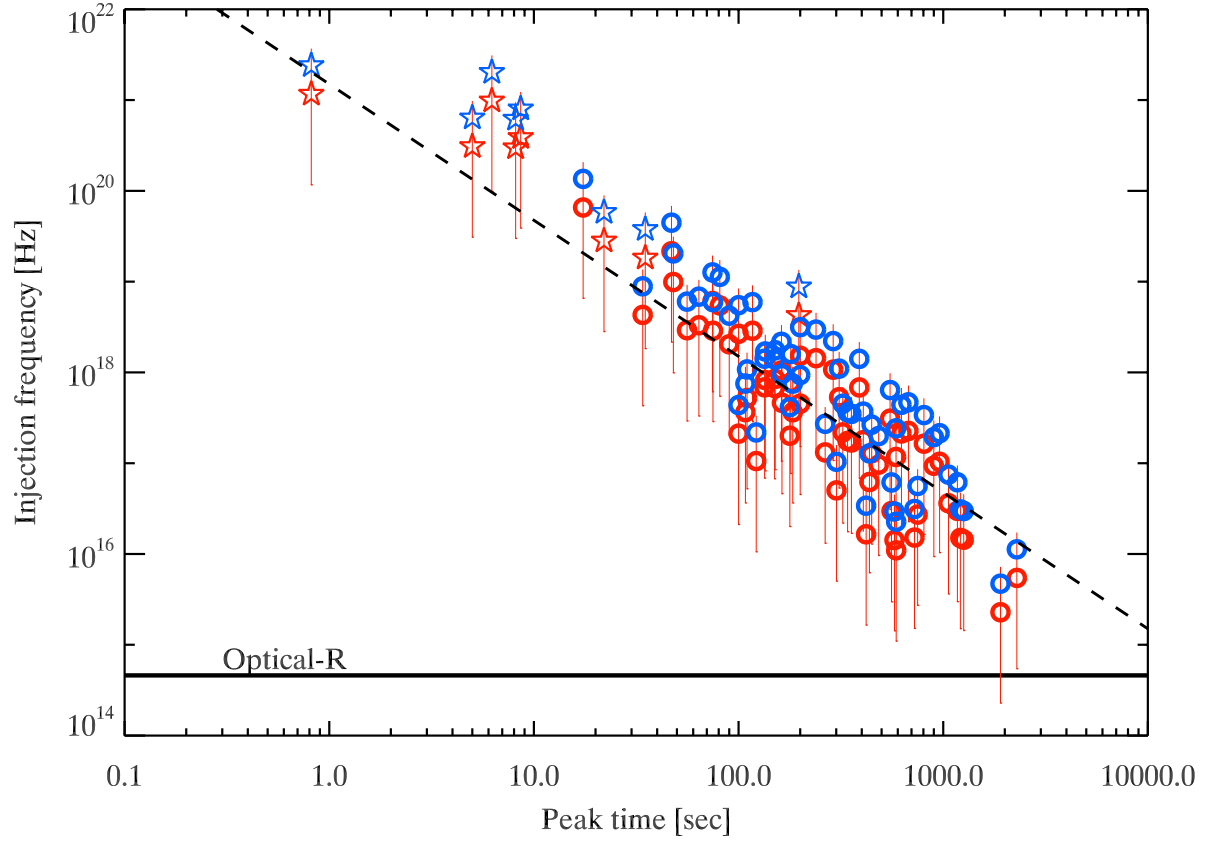

Fig. 5. Injection frequency at the $t_{\mathrm{p}}$ for each GRB. The optical-R frequency is show by the horizontal line. The injection frequency is shown for the homogeneous and wind case with red and blue symbols. GRBs with $t_{\mathrm{p}}$ from the LAT light curve are shown with star symbols. The scaling $t^{-3 / 2}$ of the injection frequency is shown for reference (it is not a fit) by the dashed line. Vertical bars, shown only for the red symbols, represent the position of the injection frequency obtained assuming $\epsilon_{\mathrm{B}}$ in the range $10^{-4}-10^{-1}$.
Molinari et al. 2007; Nava et al. 2017; Sari \& Piran 1999). The scope is to compare these methods and quantify their differences. We consider only the case of an adiabatic evolution of the fireball propagating in an external medium with a power law density profile $n(R)=n_{0} R^{-s}$. The general case of a fully radiative (and intermediate) emission regime is discussed in Nava et al. (2013). In Sect. 5 we present the results, that is, estimates of $\Gamma_{0}$, for the two popular cases $s=0$ (homogeneous medium) and $s=2$ (wind medium). The estimate and comparison of $\Gamma_{0}$ in these two scenarios for a sample of $30 \mathrm{GRBs}$ was presented, for the first time, in G12.

During the coasting phase the bulk Lorentz factor is constant ( $\Gamma=\Gamma_{0}$ ) and the bolometric light curve of the afterglow scales as $L_{\text {iso }} \propto t^{2-s}$. After the deceleration time, $\Gamma_{0}$ starts to decrease and, in the adiabatic case, the light curve scales as $L_{\text {iso }} \propto t^{-1}$ independently from the value of $s$.

Therefore, for a homogeneous medium $(s=0)$ the light curve has a peak, while in the wind case $(s=2)$ the light curve should be flat before $t_{\mathrm{p}}$ and steeper afterwards ${ }^{5} . \Gamma_{0}$ is the bulk Lorentz factor corresponding to the coasting phase. It is expected that the outflow is discontinuous with a distribution of bulk Lorentz factors (e.g. to develop internal shocks). $\Gamma_{0}$ represents the average bulk Lorentz factor of the outflow during the coasting phase.

\subsection{Sari \& Piran (1999)}

In (Sari \& Piran 1999, hereafter SP99) $\Gamma_{0}$ is derived assuming that $t_{\mathrm{p}}$ corresponds to the fireball reaching the deceleration radius $R_{\mathrm{dec}}$. This is defined as the distance from the central engine, where the mass of the interstellar medium $m\left(R_{\mathrm{dec}}\right)$, swept up by the fireball, equals $M_{0} / \Gamma_{0}$ :

$m\left(R_{\mathrm{dec}}\right)=\frac{M_{0}}{\Gamma_{0}}=\frac{E_{0}}{\Gamma_{0}^{2} c^{2}}$,

where $E_{0}$ is the isotropic equivalent kinetic energy of the fireball after the prompt phase.

\footnotetext{
5 On the other hand, as discussed in G12 and Nappo et al. (2014), there are ways to obtain a peak also in the $s=2$ case. This is why we consider both the homogeneous and the wind case for all bursts. Observationally, most early light curves indeed show a peak.
}

SP99 assume $t_{\mathrm{dec}}=R_{\mathrm{dec}} /\left(2 c \Gamma_{0}^{2}\right)$ as the link between the deceleration time $t_{\mathrm{dec}}$ and $R_{\mathrm{dec}}$. This assumption corresponds implicitly to considering that the fireball travels up to $R_{\mathrm{dec}}$ with a constant bulk Lorentz factor equal to $\Gamma_{0}$, or, in other words, that the deceleration starts instantaneously at this radius. Instead, the deceleration of the fireball starts before $R_{\mathrm{dec}}$. This approximation underestimates the deceleration time $t_{\mathrm{dec}}$ and, consequently, underestimates $\Gamma_{0}$ :

$\Gamma_{0}^{(\mathrm{SP} 99)}=\left[\left(\frac{3-s}{2^{5-s} \pi}\right)\left(\frac{E_{0}}{n_{0} m_{\mathrm{p}} c^{5-s}}\right)\right]^{\frac{1}{8-2 s}} t_{\mathrm{p}, z}^{-\frac{3-s}{8-2 s}}$,

where $t_{\mathrm{p}, z} \equiv t_{\mathrm{p}} /(1+z)$. The original formula reported in SP99 is valid only for a homogeneous medium. Here, Eq. (2) has been generalised for a generic power law density profile medium.

\subsection{Molinari et al. (2007)}

Molinari et al. (2007, hereafter M07) introduce a new formula for $\Gamma_{0}$, obtained from SP99 by modifying the assumption on $R_{\text {dec }}$. They, realistically, consider that the deceleration begins before $R_{\mathrm{dec}}$ so that $\Gamma_{\mathrm{dec}}<\Gamma_{0}$. Under this assumption, $m\left(R_{\mathrm{dec}}\right)=$ $M_{0} / \Gamma_{\mathrm{dec}}=E_{0} /\left(\Gamma_{\mathrm{dec}}^{2} c^{2}\right)$ and $t_{\mathrm{dec}}=R_{\mathrm{dec}} /\left(2 c \Gamma_{\mathrm{dec}}^{2}\right)$. However, they assume $\Gamma_{\mathrm{dec}}=\Gamma_{0} / 2$ obtaining:

$\Gamma_{0}^{(\mathrm{M} 07)}=2\left[\left(\frac{3-s}{2^{5-s} \pi}\right)\left(\frac{E_{0}}{n_{0} m_{\mathrm{p}} c^{5-s}}\right)\right]^{\frac{1}{8-2 s}} t_{\mathrm{p}, z}^{-\frac{3-s}{8-2 s}}$.

This estimate of $\Gamma_{0}$ is a factor 2 larger than that obtained by SP99: as discussed in Nava et al. (2013), M07 overestimate ${ }^{6}$ the deceleration radius by a factor $\sim 2$ and, consequently, the value of $\Gamma_{0}$ is overestimated by the same factor. This is consistent with the results of numerical one-dimensional (1D) simulations of the blast wave deceleration (Fukushima et al. 2017). Their results suggest that $\Gamma_{0}$ should be a factor $\sim 2.8$ smaller than that derived (e.g. by Liang et al. 2010) through Eq. (5).

$\overline{6}$ Liang et al. (2010) adopt the same equation as M07. 


\subsection{Ghisellini et al. (2010)}

A new method to calculate the afterglow peak time $t_{\mathrm{p}}$ is presented in (Ghisellini et al. 2010, hereafter G10). This method does not rely on the definition of the deceleration radius as in SP99 and M07. G10 derive $t_{\mathrm{p}}$ by equating the two different analytic expressions for the bolometric luminosity as a function of the time $L(t)$ during the coasting phase and during the deceleration phase ${ }^{7}$.

The relation linking the radius and the time is assumed to be: $R=2 a c t \Gamma^{2}$, where $a=1$ during the coasting phase and $a>1$ during the deceleration one. In the latter case, its value depends on the relation between the bulk Lorentz factor $\Gamma$ and the radius $R$ : for an adiabatic fireball, for instance, integration of $\mathrm{d} R=2 c \Gamma^{2} \mathrm{~d} t$, assuming $\Gamma \propto R^{-(3-s) / 2}$ according to the selfsimilar solution of Blandford \& McKee (1976, hereafter BM76), we obtain $a=4-s$.

G10 assume a relation between $\Gamma$ and $R$ which is formally identical to the BM76 solution but with a different normalisation factor:

$\Gamma(R)=\sqrt{\frac{E_{0}}{m(R) c^{2}}}=\sqrt{\frac{(3-s) E_{0}}{4 \pi n_{0} m_{\mathrm{p}} c^{2} R^{3-s}}}$,

where $m(R)$ is the interstellar mass swept by the fireball up to the radius $R$.

In G10, the authors are interested in the determination of the peak time of the light curve, but that expression can also be used to determine the initial Lorentz factor $\Gamma_{0}$. The expression, generalised for a power law profile of the external medium density, is:

$$
\Gamma_{0}^{(\mathrm{G} 10)}=\left[\left(\frac{3-s}{2^{5-s} \pi(4-s)^{3-s}}\right)\left(\frac{E_{0}}{n_{0} m_{\mathrm{p}} c^{5-s}}\right)\right]^{\frac{1}{8-2 s}} t_{\mathrm{p}, z}^{-\frac{3-s}{8-2 s}} .
$$

This estimate of $\Gamma_{0}$ is even lower than that of SP99 and, therefore, we can reasonably presume that also this value of $\Gamma_{0}$ will be underestimated with respect to the real one ${ }^{8}$.

\subsection{Ghirlanda et al. (2012)}

Ghirlanda et al. (2012) derive another formula to estimate $\Gamma_{0}$, based on the method proposed in G10, that is, intersecting the asymptotic behaviours of the bolometric light curve during the coasting phase with that during the deceleration phase. In order to describe $\Gamma(R)$ in the deceleration regime, G12 use the BM76 solution with the correct (with respect to G10) normalisation factor:

$\Gamma(R)=\sqrt{\frac{(17-4 s) E_{0}}{(12-4 s) m(R) c^{2}}}=\sqrt{\frac{(17-4 s) E_{0}}{16 \pi n_{0} m_{\mathrm{p}} c^{2} R^{3-s}}}$.

The relation between radius and time is that presented in G10:

$$
t=\left\{\begin{array}{ll}
\frac{R}{2 c \Gamma_{0}^{2}} & \text { if } t \ll t_{\mathrm{p}, z} \\
\int \frac{\mathrm{d} R}{2 c \Gamma^{2}}=\frac{R}{2(4-s) c \Gamma^{2}} & \text { if } t \gg t_{\mathrm{p}, z}
\end{array},\right.
$$

7 G10 derive $t_{\text {peak }}$ in the case of an adiabatic or a fully radiative evolution of the fireball that propagates in a homogeneous medium. For our purposes we consider only the adiabatic case.

8 Although Eq. (7) is obtained with an incorrect normalisation of the relation between $\Gamma$ and $R$, we report also this derivation since it was the first to propose a different method to derive the time of the peak of the afterglow. Cfr. with Eq. (8) showing the correct normalisation. where $t \ll t_{\mathrm{p}, z}\left(t \gg t_{\mathrm{p}, z}\right)$ corresponds to the coasting (deceleration) phase. The authors use these relations to obtain analytically the bolometric light curves before and after the peak and extrapolate them to get the intersection time which is used to infer $\Gamma_{0}$ :

$\Gamma_{0}^{(\mathrm{G} 12)}=\left[\left(\frac{17-4 s}{2^{8-s} \pi(4-s)}\right)\left(\frac{E_{0}}{n_{0} m_{\mathrm{p}} c^{5-s}}\right)\right]^{\frac{1}{8-2 s}} t_{\mathrm{p}, z}^{-\frac{3-s}{8-2 s}}$.

The main difference with respect to the formula of G10 comes from the normalisation factor of the BM76 solution and corresponds to a factor $[(17-4 s) /(12-4 s)]^{1 / 2}$.

\subsection{Nava et al. (2013)}

Nava et al. (2013, hereafter N13) propose a new model to describe the dynamic evolution of the fireball during the afterglow emission. With this model, valid for an adiabatic or full and semi-radiative regime, N13 compute the bolometric afterglow light curves and derive a new analytic formula for $\Gamma_{0}$. They rely on the same method (intersection of coasting/deceleration phase luminosity solution) already used by G10 and G12, but with a more realistic description of the dynamics of the fireball, provide an analytic formula for the estimate of $\Gamma_{0}$ in the case of a purely adiabatic evolution (Eq. (11)), and a set of numerical coefficients to be used for the full or semi-radiative evolution.

In the rest of the present work we will adopt the formula of N13 to compute $\Gamma_{0}$ so we report here their equation:

$\Gamma_{0}^{(\mathrm{N} 13)}=\left[\frac{(17-4 s)(9-2 s) 3^{2-s}}{2^{10-2 s} \pi(4-s)}\left(\frac{E_{0}}{n_{0} m_{\mathrm{p}} c^{5-s}}\right)\right]^{\frac{1}{8-2 s}} t_{\mathrm{p}, z}^{-\frac{3-s}{8-2 s}}$.

The difference with respect to the formula of G12 is due to the different relation between the shock radius $R$ and the observed time $t$. All the preceding derivations assume that most of the observed emission comes from the single point of the expanding fireball that is moving exactly toward the observer, along the line of sight. Actually, the emitted radiation that arrives at time $t$ to the observer does not come from a single point, but rather from a complex surface (Equal Arrival Time Surface, EATS) that does not coincide with the surface of the shock front. N13 avoid the rather complex computation of the EATSs, adopting a relation $t(R)$ proposed by Waxman (1997) to relate radii to times. For simplicity, in the ultra-relativistic approximation:

$t(R)=t_{\mathrm{R}}+t_{\theta}=\int\left(\frac{\mathrm{d} R}{2 \Gamma_{\mathrm{sh}}^{2} c}\right)+\frac{R}{2 \Gamma^{2} c}$,

where $\Gamma_{\mathrm{sh}}=2 \Gamma$ is the shock Lorentz factor. The time is the sum of a radial time $t_{\mathrm{R}}$, that is the delay of the shock front with respect to the light travel time at radius $R$ and an angular time $t_{\theta}$, that is the delay of photons emitted at the same radius $R$ but at larger angles with respect to the line of sight. Using the correct dynamics, N13 obtain the relation between observed time and shock front radius:

$t=\left\{\begin{array}{ll}\frac{3 R}{4 c \Gamma_{0}^{2}} & \text { if } t \ll t_{\mathrm{p}, z} \\ \frac{(9-2 s) R}{4(4-s) c \Gamma^{2}} & \text { if } t \gg t_{\mathrm{p}, z}\end{array}\right.$.

N13 use these relations to obtain analytically the bolometric light curve during the coasting and the deceleration phase and, from their intersection time, estimate $\Gamma_{0}$ through Eq. (11). 
Table 2. Comparison between the different methods of estimating $\Gamma_{0}$ found in literature.

\begin{tabular}{llrr}
\hline \hline Model & $k$ & \multicolumn{2}{c}{$\Gamma_{0} / \Gamma_{0}^{(\mathrm{N} 13)}$} \\
SP99 & {$\left[\frac{3-s}{2^{5-s} \pi}\right]^{\frac{1}{8-2 s}}$} & 0.852 & 0.772 \\
M07 & $2\left[\frac{3-s}{2^{5-s} \pi}\right]^{\frac{1}{8-2 s}}$ & 1.702 & 1.543 \\
G10 & {$\left[\frac{3-s}{2^{5-s} \pi(4-s)^{3-s}}\right]^{\frac{1}{8-2 s}}$} & 0.507 & 0.649 \\
G12 & {$\left[\frac{17-4 s}{2^{8-s} \pi(4-s)}\right]^{\frac{1}{8-2 s}}$} & & \\
N13 & {$\left[\frac{(17-4 s)(9-2 s) 3^{2-s}}{2^{10-2 s} \pi(4-s)}\right]^{\frac{1}{8-2 s}}$} & 1 & 1 \\
N14 & {$\left[\frac{(17-4 s)}{16 \pi(4-s)}\right]^{\frac{1}{8-2 s}}$} & & \\
\hline
\end{tabular}

Notes. $k$ is a dimensionless factor that varies according to the different models and the power law index of the external medium density profile $s$ (see Eq. (15)). The values of $\Gamma_{0}$ are numerically compared with the one obtained by Nava et al. (2013), in the case of homogeneous medium $(s=0)$ and wind medium $(s=2)$. We chose to compare the result of G10 using the published formula with the parameter $a=$ $4-s$ as prescribed by the Blandford \& McKee (1976) dynamics. SP99: Sari \& Piran (1999); M07: Molinari et al. (2007); G10: Ghisellini et al. (2010); G12: Ghirlanda et al. (2012); N13: Nava et al. (2013); N14: Nappo et al. (2014).

\subsection{Nappo et al. (2014)}

Nappo et al. (2014, hereafter N14) do not introduce directly a new formula to estimate $\Gamma_{0}$, but show a new way (valid only in the ultra-relativistic regime $\Gamma \gg 1$ ) to convert the shock radius $R$ into the observer time $t$, bypassing the problem of the calculation of the EATSs. They assume that most of the observed emission is produced in a ring with aperture angle $\sin \theta=1 / \Gamma$ around the line of sight. The differential form of the relation between observed time and radius can be written as $\mathrm{d} R=c \Gamma^{2} \mathrm{~d} t$, that differs from the analogous relations of $\mathrm{G} 10$ and $\mathrm{G} 12$ by a factor 2 .

Using this simplified relation coupled to the dynamics of N13 we derive a new expression for $\Gamma_{0}$ :

$\Gamma_{0}^{(\mathrm{N} 14)}=\left[\left(\frac{(17-4 s)}{16 \pi(4-s)}\right)\left(\frac{E_{0}}{n_{0} m_{\mathrm{p}} c^{5-s}}\right)\right]^{\frac{1}{8-2 s}} t_{\mathrm{p}, z}^{-\frac{3-s}{8-2 s}}$.

We show in the following paragraph that this expression provides results that are very similar to those obtained with the formula of N13, proving that the approximation on the observed times is compatible with that suggested by $\mathrm{N} 13$ and before by Waxman (1997).

\subsection{Comparison between different methods}

All the previous expressions have the same dependencies on the values of $E_{0}, n_{0}$, and $t_{\mathrm{p}, z}$. They differ only by a numeric factor and can be summarised in one single expression:

$\Gamma_{0}=k\left(\frac{E_{0}}{n_{0} m_{\mathrm{p}} c^{5-s}}\right)^{\frac{1}{8-2 s}} t_{\mathrm{p}, z}^{-\frac{3-s}{8-2 s}}$, where $k$ is a numeric factor that depends on the chosen method and on the power law index of the external medium density profile. In Table 2 we list the different values of $k$ for the various models and we show the comparison between the different estimates of $\Gamma_{0}$ for both a homogeneous and a wind medium. All the possible estimates of $\Gamma_{0}$ are within a factor $\sim 2$ of the estimate of N13; in particular the value provided by the N14 formula (Eq. (14)) is similar to N13 within a few percent.

\section{Results}

Through Eq. (11) we estimate:

- the bulk Lorentz factors $\Gamma_{0}$ of the 67 GRBs with measured $t_{\mathrm{p}}$;

- lower limits $\Gamma_{0}^{\mathrm{LL}}$ for the 85 bursts with upper limits on the onset time $t_{\mathrm{p}}^{\mathrm{UL}}$;

- upper limit $\Gamma_{0}^{\mathrm{UL}}$ for the 85 bursts with lower limits on the onset time $t_{\mathrm{p}}^{\mathrm{LL}}=T_{\mathrm{p}, \gamma}$.

We consider both a homogeneous density ISM $(s=0)$ and a wind density profile $(s=2)$. For the first case, we assume $n_{0}=1 \mathrm{~cm}^{-3}$. For the second case, $n(r)=n_{0} r^{-2}=\dot{M} / 4 \pi r^{2} m_{\mathrm{p}} v_{w}$ where $\dot{M}$ is the mass-loss rate and $v_{w}$ the wind velocity. For typical values (e.g. Chevalier \& Li 1999) $\dot{M}=10^{-5} M_{\odot} \mathrm{yr}^{-1}$ and $v_{w}=10^{3} \mathrm{~km} \mathrm{~s}^{-1}$, the normalisation of the wind case is $n_{0}=10^{35} \dot{M}_{-5} v_{w,-3}^{-1} \mathrm{~cm}^{-1}$.

In both cases we assume that the radiative efficiency of the prompt phase is $\eta=20 \%$ and estimate the kinetic energy of the blast wave in Eq. (11) as $E_{0}=E_{\text {iso }} / \eta$. The assumed typical value for $\eta$ is similar to that reported in Nava et al. (2014) and Beniamini et al. (2015) who also find a small scatter of this parameter. We notice that assuming different values of $n_{0}$ and $\eta$ within a factor of 10 and 3 with respect to those adopted in our analysis would introduce a systematic difference in the estimate of $\Gamma_{0}$ corresponding to a factor $\sim 1.5(2.3)$ for $s=0(s=2)$.

\subsection{Distribution of $\Gamma_{0}$}

Figure 6 shows the cumulative distribution of $\Gamma_{0}$. The solid red line is the distribution of $\Gamma_{0}$ for the 66 GRBs with a measure of $t_{\mathrm{p}}$. The cumulative distribution of $\Gamma_{0}^{\mathrm{LL}}$ is shown by the black dashed line (with rightward arrows) in Fig. 6. The cumulative distribution of $\Gamma_{0}^{\mathrm{UL}}$ is shown by the dotted grey line (with leftward arrows) in Fig. 6. We note that while $\Gamma_{0}^{\mathrm{LL}}$ is derived from the optical light curves decaying without any sign of the onset (i.e. providing $t_{\mathrm{p}}^{\mathrm{UL}}$ ), the limit $\Gamma_{0}^{\mathrm{UL}}$ is derived assuming that the onset time happens after the peak of the prompt emission (i.e. $\left.T_{\mathrm{p}, \gamma}\right)$.

A theoretical upper limit on $\Gamma_{0}$ can be derived from the the transparency radius, that is, $R(\tau=1)$ (Daigne \& Mochkovitch 2002). The maximum bulk Lorentz factor attainable, if the acceleration is due to the internal pressure of the fireball (i.e. $R \propto \Gamma$ ) is:

$\Gamma_{0} \leq\left(\frac{L_{\mathrm{iso}} \sigma_{T}}{8 \pi m_{\mathrm{p}} c^{3} \eta R_{0}}\right)^{1 / 4}$,

where $\sigma_{T}$ is the Thomson cross section and $R_{0}$ is the radius where the fireball is launched. We assume $R_{0} \sim 10^{8} \mathrm{~cm}$. This is consistent with the value obtained from the modelling of the photospheric emission in a few GRBs Ghirlanda et al. (2013b). The cumulative distribution of upper limits on $\Gamma_{0}$ obtained through 

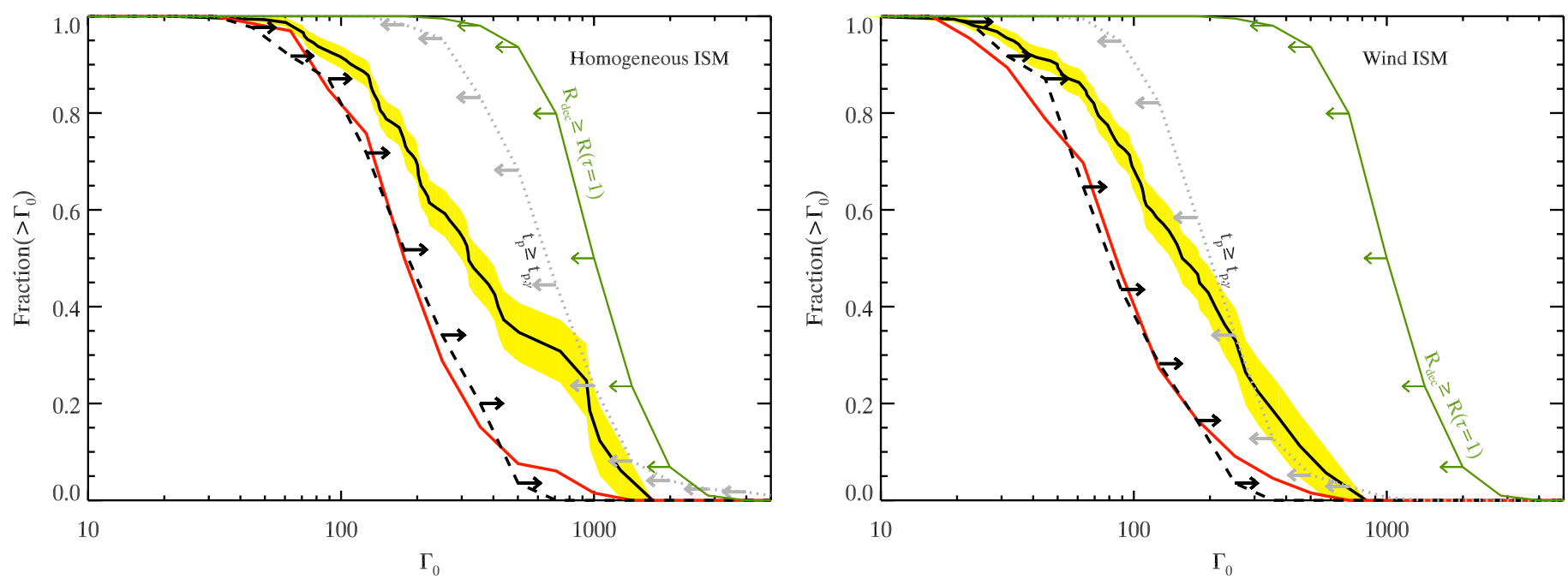

Fig. 6. Cumulative distribution of $\Gamma_{0}$ for GRBs with measured $t_{\mathrm{p}}$ (red solid line). The distribution of lower limits $\Gamma_{0}^{\mathrm{LL}}$, derived for GRBs with an upper limit on the onset time $t_{\mathrm{p}}^{\mathrm{UL}}$, is shown by the dashed black curve (with rightward arrows). Assuming $t_{\mathrm{p}} \geq T_{\mathrm{p}, \gamma}$ the distribution of upper limits on $\Gamma_{0}$ is shown by the dotted grey line (with leftward arrows). The most stringent limit on the distribution of $\Gamma_{0}$ is shown by the green solid line which assumes that deceleration radius $R_{\mathrm{dec}} \geq R(\tau=1)$, that is, the transparency radius (Eq. (16)). Joining estimates of $\Gamma_{0}$ and lower limits $\Gamma_{0}^{\mathrm{LL}}$ the reconstructed (through the KM estimator) distribution of $\Gamma_{0}$ is shown by the solid black line (and its $95 \%$ uncertainty by the yellow shaded region). The distributions are normalised to their respective number of elements. The two panels (right and left, respectively) show the case of a homogeneous $(s=0)$ and wind $(s=2)$ medium.

Table 3. Average values and $68 \%$ confidence intervals on $\Gamma_{0}$ (derived with only measured $t_{\mathrm{p}}$-red line in Fig. 6) or including lower limits $\Gamma_{0}^{\mathrm{LL}}$ (derived from upper limits $t_{\mathrm{p}}^{\mathrm{UL}}$ - black line in Fig. 6).

\begin{tabular}{lcc}
\hline \hline & $s=0[68 \%$ c.i. $]$ & $s=2[68 \%$ c.i. $]$ \\
\hline$\Gamma_{0}$ & $178[142,240]$ & $85[65,117]$ \\
$\Gamma_{0} \& \Gamma_{0}^{\mathrm{LL}}$ & $320[200,664]$ & $155[100,256]$ \\
\hline
\end{tabular}

Eq. (16), substituting for each GRB its $L_{\text {iso }}$, is shown by the green solid line in Fig. 6 (with the leftward green arrows). This distribution represents the most conservative limit on $\Gamma_{0}{ }^{9}$.

Similarly to the cumulative distributions of $t_{\mathrm{p}}$, shown in Fig. 1, also the distributions of $\Gamma_{0}$ (red solid line) and the distribution of lower limits $\Gamma_{0}^{L L}$ (black dashed line) are very close to each other. While at low and high values of $\Gamma_{0}$ the two curves are consistent with one another, for intermediate values of $\Gamma_{0}$ the distribution of lower limits is very close to that of measured $\Gamma_{0}$. In the wind case (right panel of Fig. 6), the lower limits distribution violates the distribution of measured $\Gamma_{0}$. This suggests that the distribution of $\Gamma_{0}$ obtained only with measured $t_{\mathrm{p}}$ suffers from the observational bias related to the lack of GRBs with very early optical observations.

We used the KM estimator to reconstruct the distribution of $\Gamma_{0}$, combining measurements and lower limits, similarly to what has been done in Sect. 3.1 for $t_{\mathrm{p}}$. The solid black line (with its 95\% uncertainty) in Fig. 6 shows the most likely distribution of $\Gamma_{0}$ for the population of long GRBs under the assumption of a homogeneous ISM (left panel) and for a wind medium (right panel).

The median values of $\Gamma_{0}$ (reported in Table 3) are 320 and 150 for the homogeneous and wind case, respectively, and they are consistent within their $1 \sigma$ confidence intervals. G12 found smaller average values of $\Gamma_{0}$ (i.e. 138 and 66 in the homogeneous and wind case, respectively) because of the smaller sample size

\footnotetext{
9 Changing the assumed value of $R_{0}$ within a factor of 10 shifts the green curve by a factor $\sim 1.8$.
}

(30 GRBs) and the non-inclusion of limits on $\Gamma_{0}$. Indeed, while the intermediate/small values of $\Gamma_{0}$ are reasonably well sampled by the measurements of $t_{\mathrm{p}}$, the bias against the measure of large $\Gamma_{0}$ is due to the lack of small $t_{\mathrm{p}}$ measurements (the smallest $t_{\mathrm{p}}$ are actually provided by the still few LAT detections).

The reconstructed distribution of $\Gamma_{0}$ (black line in Fig. 6) is consistent with the distribution of upper limits derived assuming $t_{\mathrm{p}} \geq T_{\mathrm{p}, \gamma}$ (dotted grey distribution) in the homogeneous case. For the wind medium there could be a fraction of GRBs $(\sim 20 \%)$ whose $t_{\mathrm{p}}$ is smaller than the peak of the prompt emission. However, Fig. 6 shows that, both in the homogeneous and wind case, the reconstructed $\Gamma_{0}$ distribution is consistent with the limiting distribution (green line) derived assuming that the deceleration occurs after transparency is reached.

\section{Correlations}

G12 found correlations between the bulk Lorentz factor $\Gamma_{0}$ and the prompt emission properties of GRBs: $L_{\text {iso }} \propto \Gamma_{0}^{2}, E_{\text {iso }} \propto \Gamma_{0}^{2}$, and with a larger scatter, $E_{\mathrm{p}} \propto \Gamma_{0}$. Interestingly, combining these correlations leads to $E_{\mathrm{p}} \propto E_{\text {iso }}^{0.5}$ and $E_{\mathrm{p}} \propto L_{\text {iso }}^{0.5}$ which are the $E_{\mathrm{p}}-E_{\text {iso }}$ (Amati et al. 2002) and Yonetoku (Yonetoku et al. 2004) correlations. G12 showed that, in order to reproduce also the $E_{\mathrm{p}}-E_{\gamma}$ correlation (Ghirlanda et al. 2007), the bulk Lorentz factor and the jet opening angle should be $\theta_{\text {jet }}^{2} \Gamma_{0}=$ const.

In this section, with the 66 long GRBs with measured $\Gamma_{0}$ (a factor $\sim 3$ larger sample than that used in G12) plus 85 lower/upper limits, we analyse the correlations of $\Gamma_{0}$ (both in the homogeneous and wind case) with $L_{\text {iso }}, E_{\text {iso }}$, and $E_{\mathrm{p}}$.

Figure 7 shows the correlation between $\Gamma_{0}$ (for the homogeneous and wind density circumburst medium, left and right panels, respectively) and $L_{\text {iso }}$. Lower limits $\Gamma_{0}^{L L}$ are shown by rightward black arrows and occupy the same region of the data points with estimated $\Gamma_{0}$ (red symbols). The green symbols show the LAT bursts which have the largest values of $L_{\text {iso }}$ and $\Gamma_{0}$. Upper limits $\Gamma_{0}^{\mathrm{UL}}$ obtained requiring that the onset of the afterglow 

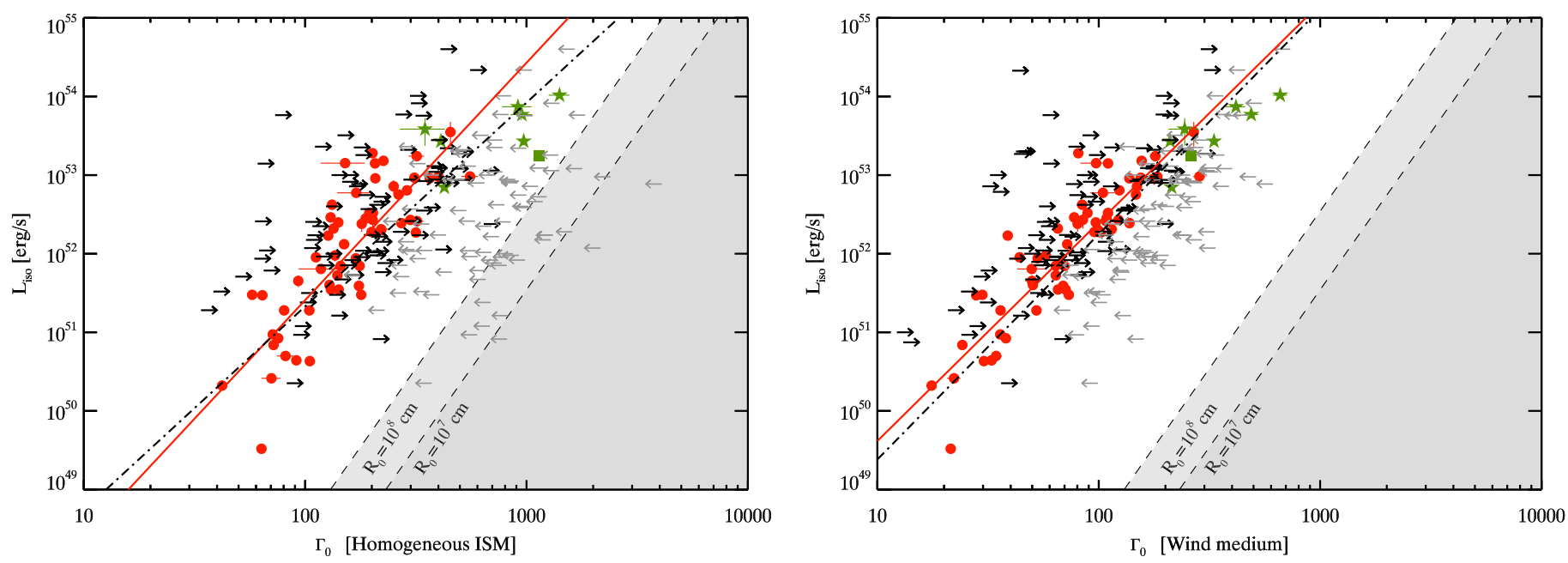

Fig. 7. Correlation between the isotropic equivalent luminosity $L_{\text {iso }}$ and the bulk Lorentz factor $\Gamma_{0}$. Estimates of $\Gamma_{0}$ from the measured afterglow onset $t_{\mathrm{p}}$ for 68 long GRBs (red filled circles and green filled stars) and the short GRB 090510 (green filled square symbol). Lower limits $\Gamma_{0}^{\mathrm{LL}}$ (derived from upper limits on the onset time $t_{\mathrm{p}}^{\mathrm{UL}}$ ) are shown by the rightward black arrows. Upper limits $\Gamma_{0}^{\mathrm{UL}}$ (derived assuming that the afterglow onset time is larger than the peak time of the prompt emission, that is, $t_{\mathrm{p}} \geq T_{\mathrm{p}, \gamma}$ ) are shown by the grey (leftward) arrows. Upper limit on $\Gamma_{0}$ imposed by the requirement that the deceleration radius is larger than the transparency radius is shown by the grey shaded triangular region (for two values of the radius $R_{0}$ where the fireball is formed - see Eq. (16)). The solid red line shows the correlation obtained with $\Gamma_{0}$ solely (bisector method), while the black dot-dashed line is the correlation obtained through the Monte Carlo method which accounts for the reconstructed distribution of $\Gamma_{0}$. Left and right panels show the case of a homogeneous and wind medium, respectively.
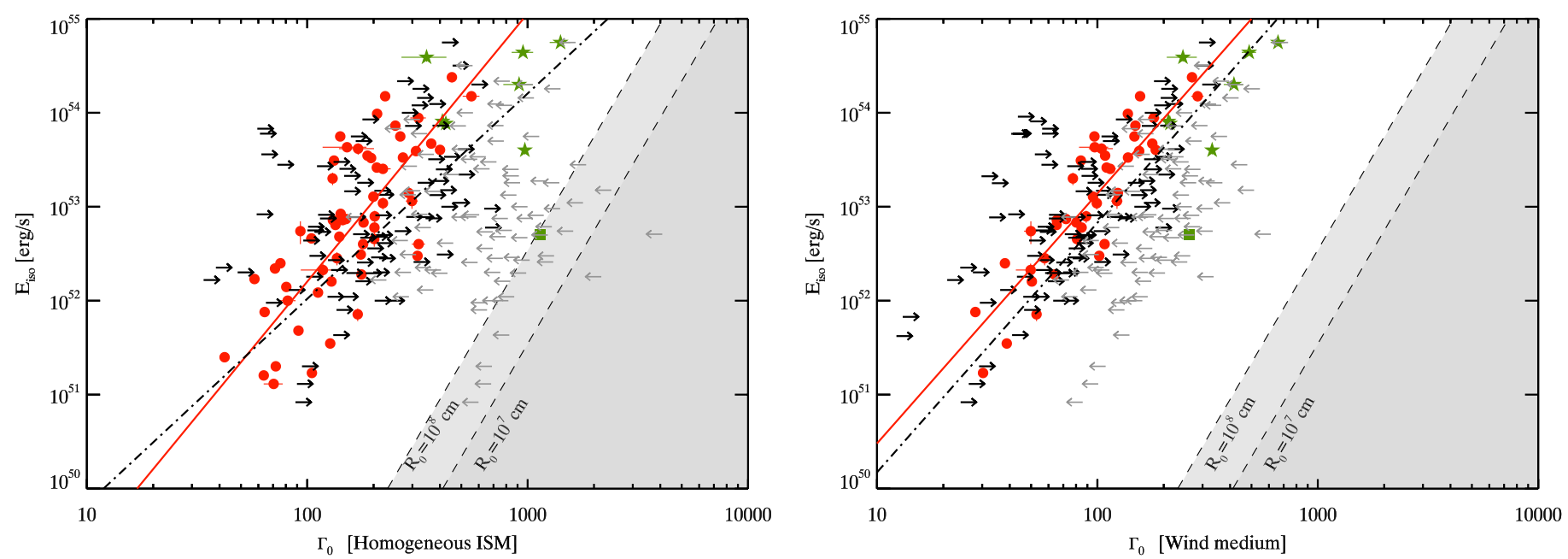

Fig. 8. Correlation between the bulk Lorentz factor $\Gamma_{0}$ and the isotropic equivalent energy $E_{\text {iso. }}$ Same symbols as Fig. 7.
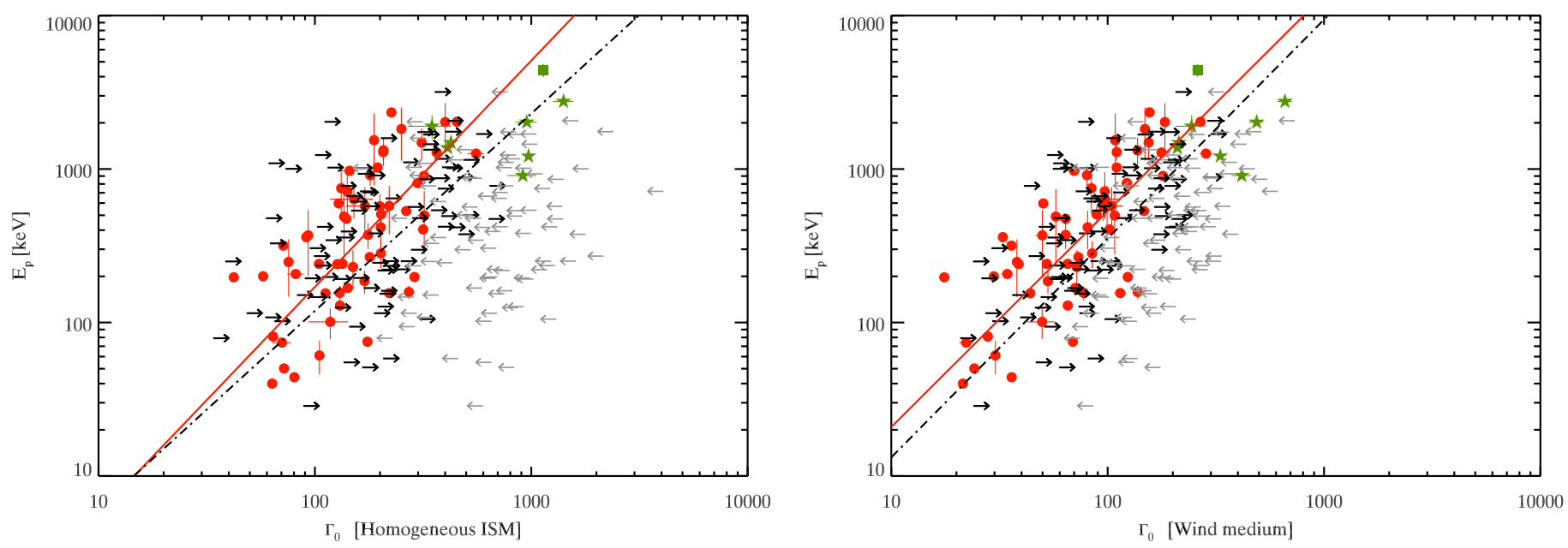

Fig. 9. Correlation between the bulk Lorentz factor $\Gamma_{0}$ and the peak energy $E_{\mathrm{p}}$. Same symbols as Fig. 7. 
Table 4. Correlations between $L_{\mathrm{iso}}, E_{\mathrm{iso}}$, and $E_{\mathrm{p}}$ with $\Gamma_{0}$.

\begin{tabular}{rcccc|cccc}
\hline \hline & \multicolumn{3}{c}{$s=0$} & \multicolumn{4}{c}{$s=2$} \\
\hline & $r$ & $P$ & $m$ & $q$ & $r$ & $P$ & $m$ & $q$ \\
\hline$L_{\text {iso }}-\Gamma_{0}$ (points) & 0.86 & $2 \times 10^{-20}$ & $3.02 \pm 0.24$ & $-0.59 \pm 0.10$ & 0.92 & $1.42 \times 10^{-27}$ & $2.78 \pm 0.17$ & $-0.40 \pm 0.05$ \\
(points+LL) & 0.62 & $1.2 \times 10^{-17}$ & $2.60 \pm 0.16$ & $-0.67 \pm 0.06$ & 0.80 & $1.9 \times 10^{-36}$ & $2.87 \pm 0.05$ & $-0.25 \pm 0.02$ \\
$E_{\text {iso }}-\Gamma_{0}$ (points) & 0.81 & $3.0 \times 10^{-16}$ & $2.85 \pm 0.22$ & $-0.20 \pm 0.10$ & 0.93 & $1.0 \times 10^{-29}$ & $2.66 \pm 0.13$ & $-1.13 \pm 0.04$ \\
(points+LL) & 0.46 & $2.8 \times 10^{-9}$ & $2.19 \pm 0.10$ & $-0.02 \pm 0.16$ & 0.73 & $1.2 \times 10^{-26}$ & $2.66 \pm 0.05$ & $-0.83 \pm 0.02$ \\
$E_{\mathrm{p}}-\Gamma_{0}$ (points) & 0.73 & $5 \times 10^{-12}$ & $1.47 \pm 0.13$ & $-0.24 \pm 0.06$ & 0.80 & $3.3 \times 10^{-16}$ & $1.41 \pm 0.11$ & $0.25 \pm 0.04$ \\
(points+LL) & 0.42 & $8 \times 10^{-8}$ & $1.28 \pm 0.03$ & $-0.40 \pm 0.03$ & 0.61 & $1 \times 10^{-16}$ & $1.43 \pm 0.03$ & $0.07 \pm 0.01$ \\
\hline
\end{tabular}

Notes. Spearman's rank correlation coefficient $r$ and associated chance probability $P$, correlation slope $m$ and normalisation $q$ for a model $Y=$ $m X+q$ (normalised according to Eqs. (17) and (18)) are reported for the homogeneous $(s=0)$ and wind $(s=2)$ case. For each correlation the results considering only GRBs with estimated $\Gamma_{0}$ and including lower limits $\Gamma_{0}^{\mathrm{LL}}$ are given.

happens after the main emission peak of the prompt light curve are shown by the grey (leftward) arrows.

The shadowed grey region shown in Fig. 7 shows the limit obtained requiring that the deceleration happens after the transparency radius, that is, $R_{\mathrm{dec}} \geq R_{\mathrm{th}}$. Two different limits are shown corresponding to different (by a factor of 10) assumptions for $R_{0}$, that is, the radius where the jet is launched (see Eq. (16)). The correlation between $L_{\text {iso }}$ and $\Gamma_{0}$ in the wind profile (bottom panel of Fig. 7) is less scattered than in the homogeneous case (upper panel of Fig. 7). The correlations between $\Gamma_{0}$ and the isotropic energy $E_{\text {iso }}$ and the peak energy $E_{\mathrm{p}}$ are shown in Figs. 8 and 9, respectively. Symbols are the same as in Fig. 7. Also in these cases, the correlation in the wind case is less scattered than in the homogeneous case.

The correlations shown in Figs. 7-9 were analysed computing the Spearman's rank correlation coefficient $r$ and its probability $P$ and fitting the data, with the bisector method, with a linear model:

$\log \left(\frac{Y}{10^{52} \text { erg }}\right)=m \log \left(\frac{\Gamma_{0}}{100}\right)+q$,

where $Y$ is either $L_{\text {iso }}$ or $E_{\text {iso }}$. Similarly, for the correlation between $E_{\mathrm{p}}$ and $\Gamma_{0}$ we adopted the model:

$\log \left(\frac{E_{\text {peak }}}{300 \mathrm{keV}}\right)=m \log \left(\frac{\Gamma_{0}}{100}\right)+q$.

The results are given in Table 4 both for the homogeneous and for the wind case. Correlation analysis considering only estimates of $\Gamma_{0}$ are shown by the red solid line in Figs. 7-9.

In order to reconstruct the correlation considering measured $\Gamma_{0}$ and upper/lower limits, we adopted the same Monte Carlo procedure described in Sect. 3. We generate $10^{5}$ samples composed by the GRBs with measured $\Gamma_{0}$ and assigning to the 85 GRBs without measured $t_{\mathrm{p}}$ a value of $\Gamma_{0}$ randomly extracted from the reconstructed distribution of $\Gamma_{0}$ shown in Fig. 6 . We then analyse the correlations of $\Gamma_{0}$ and the energetic variables within these random samples and report the central values of the correlation parameters (coefficient, probability, slope and normalisation) in Table 4. In all cases we find significant correlations also when upper/lower limits are accounted for with this Monte Carlo method. The corresponding correlation lines are shown with the dot-dashed lines in Figs. 7-9. We note that the correlations found with only $\Gamma_{0}$ or reconstructed accounting also for limits are very similar.

Figure 7 (and similarly Figs. 8 and 9) shows that the planes are not uniformly filled with data points, contrary to what is claimed by Hascoët et al. (2014). Indeed, the right part of the planes of Figs. 7 and 8 corresponding to large $\Gamma_{0}$ and any possible value of $L_{\text {iso }}$ and $E_{\text {iso }}$, respectively, are limited by the excluded region, that is, deceleration should happen after the fireball transparency. Moreover, the upper limits obtained requiring that the afterglow onset time $t_{\mathrm{p}}$ is after the main prompt emission peak leads to the upper limits shown by the grey downward arrows in Figs. 7 and 8, which are even more constraining than the shaded regions. This confirms that the bulk Lorentz factor is indeed strongly correlated with the prompt emission properties $\left(E_{\text {iso }}, L_{\text {iso }}\right.$, and $\left.E_{\mathrm{p}}\right)$.

We compute the comoving frame peak energy and isotropic energy with the equations derived in G12: $E_{\text {peak }}^{\prime}=3 E_{\text {peak }} / 5 \Gamma_{0}$ and $E_{\text {iso }}^{\prime}=E_{\text {iso }} / \Gamma_{0}$. The luminosity $L_{\text {iso }}^{\prime}=3 L_{\text {iso }} / 4 \Gamma_{0}^{2}$. Primed quantities refer to the comoving frame. For GRBs with a $t_{\mathrm{p}}^{\mathrm{UL}}$ a lower limit $\Gamma_{0}^{\mathrm{LL}}$ transforms into an upper limit on $L_{\mathrm{iso}}^{\prime}, E_{\mathrm{iso}}^{\prime}$, and $E_{\mathrm{p}}^{\prime}$. Vice versa, lower limits $t_{\mathrm{p}}^{\mathrm{LL}}$ provide upper limits $\Gamma_{0}^{\mathrm{UL}}$ which transform the rest frame observables into lower limits in the comoving frame. Through the Monte Carlo method adopted in the previous sections we derive the distributions of the comoving frame $E_{\text {iso }}^{\prime}, L_{\text {iso }}^{\prime}$, and $E_{\mathrm{p}}^{\prime}$; these are shown in Fig. 10 . The average values of $\Gamma_{0}$ for the homogeneous ISM is larger than that of the wind case. For this reason the distributions of the comoving frame quantities shown in Fig. 10 are slightly shifted in the two scenarios with the homogeneous case resulting in a slightly smaller average value of the comoving frame isotropic energy, luminosity, and peak energy. The average values of the comoving frame distributions shown in Fig. 10 are reported in Table 5.

\section{Summary}

The present work assembles the largest sample of $t_{\mathrm{p}}$, by revising and expanding (to June 2016) the original sample of G12, and including upper limits $t_{\mathrm{p}}^{\mathrm{UL}}$ corresponding to GRBs without a measured onset time. Our sample, presented in Table A.1, is composed of:

- 67 GRBs with measured $t_{\mathrm{p}}$ : 66 long and one short (GRB 090510). Eight $t_{\mathrm{p}}$ are measured from the $\mathrm{GeV}$ light curve.

- 106 long GRBs with an upper limit $t_{\mathrm{p}}^{\mathrm{UL}}$. These are GRBs detected in the optical or GeV band within one day and showing a decaying light curve. Five $t_{\mathrm{p}}^{\mathrm{UL}}$ are measured from the GeV light curve. 

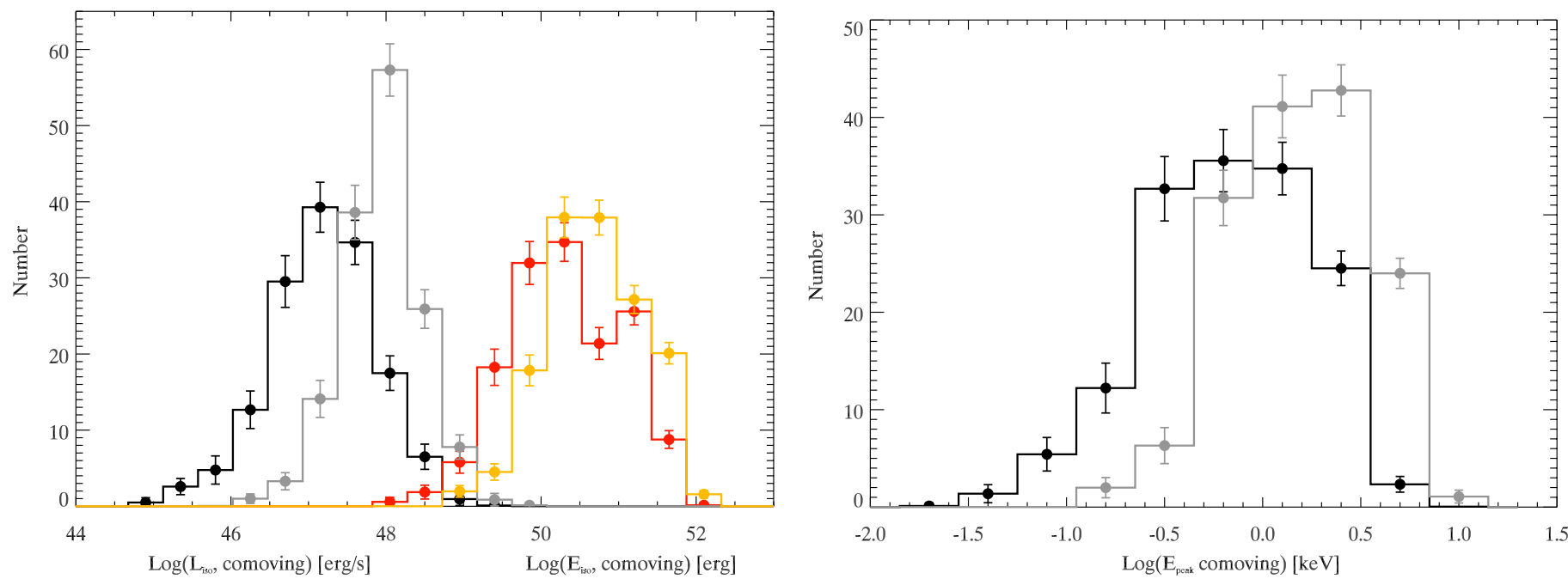

Fig. 10. Distribution of comoving frame properties of GRBs. Left panel: comoving frame isotropic luminosity (back and grey histogram for the homogeneous and wind case, respectively) and of the isotropic energy (red and orange histogram for the homogeneous and wind case, respectively). Right panel: comoving frame peak energy (black and grey histogram for the homogeneous and wind case, respectively).

Table 5. Average values and width of the distribution of the (log values of) $E_{\mathrm{iso}}, L_{\mathrm{iso}}$, and $E_{\mathrm{p}}$, in the rest frame and in the comoving frame (both for the homogeneous and the wind density profile).

\begin{tabular}{lc|cc}
\hline \hline & Rest frame & \multicolumn{2}{|c}{ Comoving frame } \\
& & $s=0$ & $s=2$ \\
& Median [68\% c.i.] & Median [68\% c.i.] & Median [68\% c.i.] \\
\hline Isotropic energy [erg] & $52.88[52.25,53.74]$ & $50.50[49.85,51.33]$ & $50.89[50.33,51.57]$ \\
Isotropic luminosity [erg s $\left.{ }^{-1}\right]$ & $52.40[51.60,53.16]$ & $47.43[46.79,48.03]$ & $48.18[47.70,48.63]$ \\
Peak energy [keV] & $2.64[2.18,3.08]$ & $-0.008[-0.42,0.43]$ & $0.35[0.02,0.69]$ \\
\hline
\end{tabular}

An upper limit $t_{\mathrm{p}}^{\mathrm{UL}}$ gives a lower limit $\Gamma_{0}^{\mathrm{LL}}$ according to equations in Sect. 4. Accounting only for the most stringent upper limits, we consider only the 85 bursts with $t_{\mathrm{p}}^{\mathrm{UL}} \lesssim 11500 \mathrm{~s}$, corresponding to five times the maximum $t_{\mathrm{p}}$ among the 66 GRBs. Therefore, the final sample is composed of 151 GRBs: 66 long GRBs ${ }^{10}$ with $t_{\mathrm{p}}$ and 85 GRBs with $t_{\mathrm{p}}^{\mathrm{UL}}$.

The observable is $t_{\mathrm{p}}$ : Fig. 1 shows that the relative position of the cumulative distribution of $t_{\mathrm{p}}$ (red line) and of lower limits $t_{\mathrm{p}}^{\mathrm{UL}}$ (dashed line) suggests the presence of a selection bias against the measurement of intermediate/small $t_{\mathrm{p}}$ values. The earliest $t_{\mathrm{p}}$ are provided by the few GRBs with an onset measured from the GeV light curve by the LAT on board Fermi.

We can extract more information from the sample of $t_{\mathrm{p}}$ if we use also upper limits $t_{\mathrm{p}}^{\mathrm{UL}}$. Statistical methods (e.g. Feigelson \& Nelson 1985) allow us to reconstruct the distribution of an observable adopting measurements and upper limits, provided upper limits cover the same range of values of detections. This is our case as shown in Fig. 1. We adopt the KaplanMeier estimator to reconstruct the distribution of $t_{\mathrm{p}}$ of the population of long GRBs. We find that:

1. The reconstructed distribution of $t_{\mathrm{p}}$ (solid black line with yellow shaded region in Fig. 1) has median value $\left\langle t_{\mathrm{p}}\right\rangle \sim 60 \mathrm{~s}$ extending from a few seconds $\left(t_{\mathrm{p}}\right.$ from LAT) to relatively late $t_{\mathrm{p}} \sim 10^{3} \mathrm{~s}$; in the rest frame, the average $t_{\mathrm{p}} /(1+z)$ is $20 \mathrm{~s}$. ${ }^{10}$ In most of the Figures we show for comparison also the short
GRB 090510: this is not included in the quantitive analysis.
2. The $t_{\mathrm{p}}$ distribution is consistent with the cumulative distribution of $T_{\mathrm{p}, \gamma}$ (dot-dashed grey line in Fig. 1). $T_{\mathrm{p}, \gamma}$ is the time when the prompt emission light curve peaks.

3 . The rest frame $t_{\mathrm{p}} /(1+z)$ is inversely correlated with $E_{\text {iso }}, L_{\text {iso }}$ and $E_{\mathrm{p}}$ (Fig. 4). These correlations are statistically significant (Table 1).

Since $\Gamma_{0} \propto t_{\mathrm{p}}^{-(3-s) /(8-2 s)}$, an upper limit on $t_{\mathrm{p}}$ provides a lower limit $\Gamma_{0}^{\mathrm{LL}}$. We combine $\Gamma_{0}$ and $\Gamma_{0}^{\mathrm{LL}}$ finding that:

4. The reconstructed distribution of $\Gamma_{0}$ (solid black line and yellow shaded region in Fig. 6) has a median of $\left\langle\Gamma_{0}\right\rangle=320$ and 155 in the homogeneous and wind case, respectively (Table 3).

5. $\Gamma_{0}$ values span two orders of magnitude from 20 to 1000 in the wind case (right panel in Fig. 6) and a slightly smaller range in the homogeneous case (left panel in Fig. 6).

6. The distribution of $\Gamma_{0}$ is consistent with the distribution of upper limits derived under the assumption that the afterglow peak is larger than the peak of the prompt emission (i.e. $t_{\mathrm{p}}>$ $T_{\mathrm{p}, \gamma}$ - dotted grey line in Fig. 6).

Through a Monte Carlo method we combine the $66 t_{\mathrm{p}}$ with the $85 \mathrm{GRBs}$ with $t_{\mathrm{p}}^{\mathrm{UL}}$. In the latter cases $\Gamma_{0}$ should lie in the range $\Gamma_{0}^{\mathrm{LL}}<\Gamma_{0}<\Gamma_{0}^{\mathrm{UL}}$ (where $\Gamma_{0}^{\mathrm{LL}}$ and $\Gamma_{0}^{\mathrm{UL}}$ are obtained from $t_{\mathrm{p}}^{\mathrm{UL}}$ and $T_{\mathrm{p}, \gamma}$ ). We assign values of $\Gamma_{0}$, randomly extracted from the reconstructed distribution and requiring they are comprised within the above limits, to the 85 GRBs. Creating $10^{5}$ mock samples of 


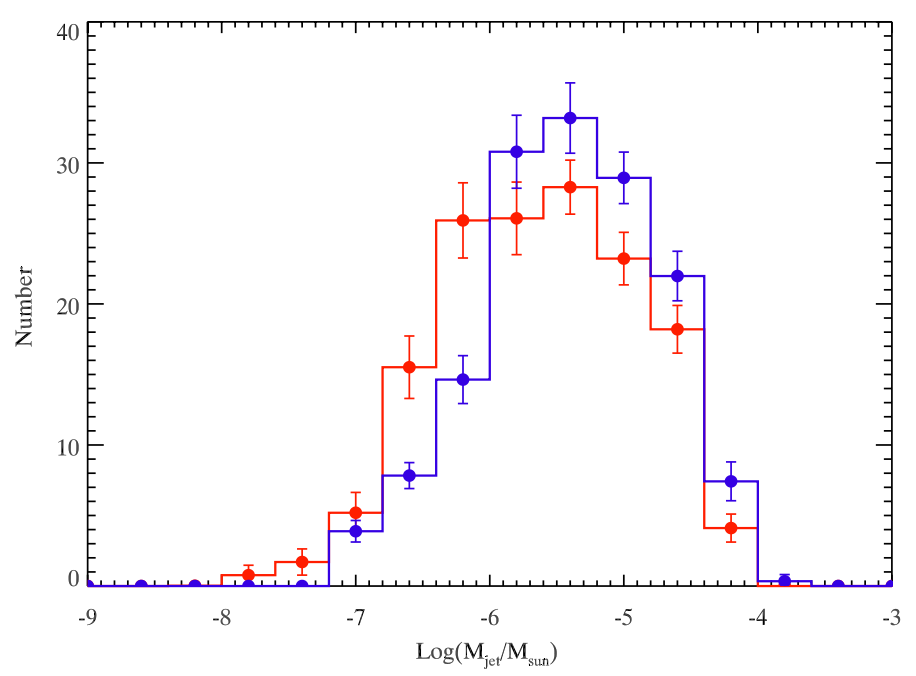

Fig. 11. Distribution of the mass of the jet (in solar masses) for the homogeneous case (red solid line) and for the wind case (blue solid line) computed assuming a typical jet opening angle of 5 degrees.

66 GRBs with measured $\Gamma_{0}$ plus the randomly assigned 85 values, we evaluate the various dependencies of this parameter on the prompt emission properties analysing these random samples:

1. There are significant correlations between $\Gamma_{0}$ and the prompt emission rest frame properties, namely $E_{\text {iso }}, L_{\text {iso }}$, and $E_{\mathrm{p}}$ (shown in Figs. 7, 8, and 10 and Table 4):

$$
\begin{aligned}
& L_{\text {iso }} \propto \Gamma_{0, \mathrm{H}}^{2.6} \quad L_{\text {iso }} \propto \Gamma_{0, \mathrm{~W}}^{2.9}, \\
& E_{\text {iso }} \propto \Gamma_{0, \mathrm{H}}^{2.2} \quad E_{\text {iso }} \propto \Gamma_{0, \mathrm{~W}}^{2.7}, \\
& E_{\mathrm{p}} \propto \Gamma_{0, \mathrm{H}}^{1.3} \quad E_{\mathrm{p}} \propto \Gamma_{0, \mathrm{~W}}^{1.4},
\end{aligned}
$$

where $\mathrm{H}$ and $\mathrm{W}$ denote the homogeneous and wind case, respectively. The correlations are less scattered in the $\mathrm{W}$ case.

2. The distribution of the data points and upper/lower limits in Figs. 7-9 show that these planes are not uniformly filled with data.

3. There is no correlation between the rest frame duration $T_{90} /(1+z)$ and $\Gamma_{0}$.

With the distribution of bulk Lorentz factors reconstructed from our sample of GRBs, we can compute the baryon loading of the fireball $M=E_{\mathrm{k}} / \Gamma_{0} c^{2}$, where $E_{\mathrm{k}}=E_{\mathrm{iso}} / \eta$ is the kinetic energy of the blast wave. This would lead to the isotropic equivalent mass loading but, since GRBs have a jet, we derive the baryon loading of the jet assuming a typical opening angle of 5 degrees (e.g. Ghirlanda et al. 2007). Figure 11 shows the distribution of the jet baryon loading in units of solar masses obtained in the homogeneous (red line) and wind (blue line) case. The baryon loading is distributed around a typical value of a few $10^{-6} M_{\odot}$ which is similar in the two density scenarios.

\section{Conclusions}

We have extended the sample of GRBs with measured onset time $t_{\mathrm{p}}$ including for the first time also upper limits $t_{\mathrm{p}}$. While the onset time happens relatively early (the mean value of the distribution, accounting for upper limits, is $\sim 60 \mathrm{~s}$ in the observer frame), in most cases this happens after the time of the peak of the prompt emission of GRBs. This ensures that a considerable fraction of the fireball kinetic energy should have been transferred to the blast wave whose deceleration produces $t_{\mathrm{p}}$. Therefore, the so called "thin shell approximation" (i.e. the requirement that most of the fireball energy has been transferred to the blast wave that decelerates into the interstellar medium) adopted in deriving $\Gamma_{0}$ from the measure of $t_{\mathrm{p}}$ holds. This regime is only partly established in jets that remain highly magnetised at the afterglow stage (Mimica et al. 2009) and seems to support a low magnetisation outflow. Moreover, we have shown that the synchrotron characteristic frequency $v_{\text {inj }}$, computed at $t_{\mathrm{p}}$, is above the optical $R$ band (Fig. 5) for all bursts. This ensures that it cannot be responsible for the peak of the afterglow light curve sweeping through the observer frame optical band. This allows us to interpret $t_{\mathrm{p}}$ as the fireball deceleration onset time and use it to compute the bulk Lorentz factor $\Gamma_{0}$.

We have reviewed and compared the different methods and formulae proposed in the literature (Ghirlanda et al. 2012; Ghisellini et al. 2010; Molinari et al. 2007; Nappo et al. 2014; Nava et al. 2013; Sari \& Piran 1999) to compute $\Gamma_{0}$ from $t_{\mathrm{p}}$. They differ at most by a factor of two. Therefore, any different choice of the specific formula to compute $\Gamma_{0}$ should only introduce a small systematic difference in the derived correlation normalisation and average values.

The average $\Gamma_{0}$ is 320 and 155, for the homogeneous and wind cases, respectively. These values are larger than those found in G12 due to our larger sample (almost a factor 2) and to the inclusion of lower limits $\Gamma_{0}^{\mathrm{LL}}$. We confirm the existence of significant correlations between the GRB bulk Lorentz factor and the prompt emission observables, that is, isotropic energy and luminosity and peak energy (Eq. (21)). The $\Gamma_{0}-L_{\text {iso }}, \Gamma_{0}-$ $E_{\text {iso }}$, and $\Gamma_{0}-E_{\mathrm{p}}$ correlations are not boundaries in these planes (opposite to what claimed by Hascoët et al. 2014). With respect to the sample considered in Hascoët et al. (2014), our samples of $t_{\mathrm{p}}$ and $t_{\mathrm{p}}^{\mathrm{UL}}$ are a factor three and four larger, respectively.

By combining these correlations, we find $E_{\mathrm{p}} \propto E_{\text {iso }}^{0.58}$ and $E_{\mathrm{p}} \propto L_{\text {iso }}^{0.50}$ in the homogenous case (and $E_{\mathrm{p}} \propto E_{\text {iso }}^{0.53}$ and $E_{\mathrm{p}} \propto L_{\text {iso }}^{0.50}$ in the wind case). These slopes are consistent with the $E_{\mathrm{p}}-E_{\text {iso }}$ and $E_{\mathrm{p}}-L_{\text {iso }}$ correlations (Amati et al. 2002; Yonetoku et al. 2004) that we have re-derived here through our sample of $151 \mathrm{GRBs}$, and are $E_{\mathrm{p}} \propto E_{\text {iso }}^{0.55 \pm 0.03}$ and $E_{\mathrm{p}} \propto L_{\text {iso }}^{0.53 \pm 0.03}$, respectively.

Finally, the knowledge of $\Gamma_{0}$ allows us to derive the mass of the fireball for individual GRBs. Assuming a typical jet opening angle of 5 degrees, the jet mass $\mathbf{M}_{\text {jet }}$ is similarly distributed for the homogeneous and wind cases (red and blue lines, respectively, in Fig. 11) between $10^{-8}$ and $10^{-4} M_{\odot}$.

Acknowledgements. INAF PRIN 2014 (1.05.01.94.12) is acknowledged. The anonymous referee is acknowledged for useful comments that helped to improve the content of the paper.

\section{References}

Abdo, A. A., Ackermann, M., Arimoto, M., et al. 2009a, Science, 323, 1688 Abdo, A. A., Ackermann, M., Asano, K., et al. 2009b, ApJ, 707, 580 Ackermann, M., Ajello, M., Baldini, L., et al. 2010a, ApJ, 717, L127 Ackermann, M., Asano, K., Atwood, W. B., et al. 2010b, ApJ, 716, 1178 Ackermann, M., Ajello, M., Asano, K., et al. 2013, ApJS, 209, 11 Ackermann, M., Ajello, M., Asano, K., et al. 2014, Science, 343, 42 Akerlof, C. W., Kehoe, R. L., McKay, T. A., et al. 2003, PASP, 115, 132 Amati, L., Frontera, F., Tavani, M., et al. 2002, A\&A, 390, 81 Bardho, O., Gendre, B., Rossi, A., et al. 2016, MNRAS, 459, 508 Baring, M. G., \& Harding, A. K. 1997, ApJ, 491, 663

Beniamini, P., \& van der Horst, A. J. 2017, MNRAS, 472, 3161 Beniamini, P., Nava, L., Duran, R. B., \& Piran, T. 2015, MNRAS, 454, 1073 
Beniamini, P., Nava, L., \& Piran, T. 2016, MNRAS, 461, 51

Berger, E., Kulkarni, S. R., Bloom, J. S., et al. 2002, ApJ, 581, 981

Bhalerao, V. B., Singer, L. P., Kasliwal, M. M., et al. 2014, GRB Coordinates Network, 16442

Blandford, R. D., \& McKee, C. F. 1976, Phys. Fluids, 19, 1130

Bloom, J. S., Perley, D. A., Li, W., et al. 2009, ApJ, 691, 723

Breeveld, A. A., \& Kuin, N. P. M. 2013, GRB Coordinates Network, 14957

Buckley, D., Potter, S., Kniazev, A., et al. 2014, GRB Coordinates Network, 17237

Burd, A., Cwiok, M., Czyrkowski, H., et al. 2005, New Astron., 10, 409

Burgess, J. M., Bégué, D., Ryde, F., et al. 2016, ApJ, 822, 63

Butler, N. R., Kocevski, D., Bloom, J. S., \& Curtis, J. L. 2007, ApJ, 671, 656

Butler, N. R., Bloom, J. S., \& Poznanski, D. 2010, ApJ, 711, 495

Cenko, S. B., \& Perley, D. A. 2015, GRB Coordinates Network, 17612

Cenko, S. B., Urban, A. L., Perley, D. A., et al. 2015, ApJ, 803, L24

Covino, S., D'Avanzo, P., Klotz, A., et al. 2008, MNRAS, 388, 347

Cucchiara, A., Cenko, S. B., Bloom, J. S., et al. 2011, ApJ, 743, 154

Daigne, F., \& Mochkovitch, R. 2002, MNRAS, 336, 1271

de Pasquale, M., \& Cannizzo, J. K. 2013, GRB Coordinates Network, 14573

Denisenko, D., Gorbovskoy, E., Lipunov, V., et al. 2012, GRB Coordinates Network, 13623

Diercks, A. H., Deutsch, E. W., Castander, F. J., et al. 1998, ApJ, 503, L105

Feigelson, E. D., \& Nelson, P. I. 1985, ApJ, 293, 192

Fenimore, E. E., Epstein, R. I., \& Ho, C. 1993, A\&AS, 97, 59

Ferrero, A., Hanlon, L., Felletti, R., et al. 2010, Adv. Astron., 2010, 715237

Filgas, R., Greiner, J., Schady, P., et al. 2012, A\&A, 546, A101

Frail, D. A., Kulkarni, S. R., Nicastro, L., Feroci, M., \& Taylor, G. B. 1997, Nature, 389, 261

Fukushima, T., To, S., Asano, K., \& Fujita, Y. 2017, ApJ, 844, 92

Fynbo, J. U., Gorosabel, J., Dall, T. H., et al. 2001, A\&A, 373, 796

Galama, T. J., Briggs, M. S., Wijers, R. A. M. J., et al. 1999, Nature, 398, 394

Gendre, B., Klotz, A., Palazzi, E., et al. 2010, MNRAS, 405, 2372

Genet, F., Daigne, F., \& Mochkovitch, R. 2007, MNRAS, 381, 732

Ghirlanda, G., Nava, L., Ghisellini, G., \& Firmani, C. 2007, A\&A, 466, 127

Ghirlanda, G., Ghisellini, G., \& Nava, L. 2010, A\&A, 510, L7

Ghirlanda, G., Nava, L., Ghisellini, G., et al. 2012, MNRAS, 420, 483

Ghirlanda, G., Ghisellini, G., Salvaterra, R., et al. 2013a, MNRAS, 428, 1410

Ghirlanda, G., Pescalli, A., \& Ghisellini, G. 2013b, MNRAS, 432, 3237

Ghisellini, G., Ghirlanda, G., Nava, L., \& Firmani, C. 2007, ApJ, 658, L75

Ghisellini, G., Nardini, M., Ghirlanda, G., \& Celotti, A. 2009, MNRAS, 393, 253

Ghisellini, G., Ghirlanda, G., Nava, L., \& Celotti, A. 2010, MNRAS, 403, 926

Goodman, J. 1986, ApJ, 308, L47

Gorbovskoy, E., Krushinski, V., Pruzhinskaya, M., et al. 2014a, GRB Coordinates Network, 16500

Gorbovskoy, E., Lipunov, V., Kornilov, V., et al. 2014b, GRB Coordinates Network, 16307

Gorbovskoy, E., Lipunov, V., Pruzhinskaya, M., et al. 2014c, GRB Coordinates Network, 15932

Gorbovskoy, E. S., Lipunov, V. M., Buckley, D. A. H., et al. 2016, MNRAS, 455,3312

Gorosabel, J., Mottola, S., de Ugarte Postigo, A., Hellmich, S., \& Proffe, G. 2013, GRB Coordinates Network, 15469

Gorosabel, J., Hellmich, S., de Ugarte Postigo, A., Mottola, S., \& Thoene, C. 2014, GRB Coordinates Network, 17197

Graff, P., Feroz, F., Hobson, M. P., \& Lasenby, A. 2014, MNRAS, 441, 1741

Granot, J., \& van der Horst, A. J. 2014, PASA, 31, e008

Greiner, J., Krühler, T., Fynbo, J. P. U., et al. 2009, ApJ, 693, 1610

Groot, P. J., Galama, T. J., Vreeswijk, P. M., et al. 1998, ApJ, 502, L123

Grupe, D., Brown, P. J., Cummings, J., et al. 2006, ApJ, 645, 464

Guidorzi, C., Steele, I. A., Melandri, A., et al. 2009, GRB Coordinates Network, 9375

Halpern, J. P., Uglesich, R., Mirabal, N., et al. 2000, ApJ, 543, 697

Hascoët, R., Daigne, F., Mochkovitch, R., \& Vennin, V. 2012, MNRAS, 421 525

Hascoët, R., Beloborodov, A. M., Daigne, F., \& Mochkovitch, R. 2014, ApJ, 782,5

Henden, A., Gross, J., Denny, B., Terrell, D., \& Cooney, W. 2009, GRB Coordinates Network, 9211

Holland, S., Fynbo, J. P. U., Hjorth, J., et al. 2001, A\&A, 371, 52

Holland, S. T., Soszyński, I., Gladders, M. D., et al. 2002, AJ, 124, 639

Ioka, K., Toma, K., Yamazaki, R., \& Nakamura, T. 2006, A\&A, 458, 7

Isobe, T., Feigelson, E. D., \& Nelson, P. I. 1986, ApJ, 306, 490

Isobe, T., Feigelson, E. D., Akritas, M. G., \& Babu, G. J. 1990, ApJ, 364, 104

Japelj, J., Kopac, D., Guidorzi, C., Mundell, C., \& Virgili, F. 2012, GRB Coordinates Network, 14058

Japelj, J., Kopač, D., Kobayashi, S., et al. 2014, ApJ, 785, 84

Jin, Z.-P., Covino, S., Della Valle, M., et al. 2013, ApJ, 774, 114
Kasliwal, M. M., Cenko, S. B., \& Singer, L. P. 2014, GRB Coordinates Network, 16425

King, O. G., Blinov, D., Giannios, D., et al. 2014, MNRAS, 445, L114

Kinugasa, K., \& Torii, K. 2004, GRB Coordinates Network, 2832

Klotz, A., Boër, M., Atteia, J. L., \& Gendre, B. 2009, AJ, 137, 4100

Klotz, A., Turpin, D., Atteia, J. L., et al. 2016, GRB Coordinates Network, 19622

Krimm, H. A., Granot, J., Marshall, F. E., et al. 2007, ApJ, 665, 554

Krolik, J. H., \& Pier, E. A. 1991, ApJ, 373, 277

Kruehler, T., Malesani, D., Xu, D., et al. 2014, GRB Coordinates Network, 15899

Krühler, T., Ledoux, C., Fynbo, J. P. U., et al. 2013, A\&A, 557, A18

Kuin, N. P. M., \& Yershov, V. N. 2012, GRB Coordinates Network, 13808

Kuin, P., de Pasquale, M., Malesani, D., \& Brown, P. 2015, GRB Coordinates Network, 18224

Kumar, P., \& Barniol Duran, R. 2010, MNRAS, 409, 226

Kumar, P., \& Zhang, B. 2015, Phys. Rep., 561, 1

Lacluyze, A., Haislip, J., Ivarsen, K., et al. 2011, GRB Coordinates Network, 12535

Laskar, T., Alexander, K. D., Berger, E., et al. 2016, ApJ, 833, 88

Lei, W.-H., Zhang, B., \& Liang, E.-W. 2013, ApJ, 765, 125

Li, W., Filippenko, A. V., Chornock, R., \& Jha, S. 2003, ApJ, 586, L9

Liang, E.-W., Yi, S.-X., Zhang, J., et al. 2010, ApJ, 725, 2209

Liang, E.-W., Li, L., Gao, H., et al. 2013, ApJ, 774, 13

Lien, A. Y., Barthelmy, S., Maselli, A., \& Oates, S. R. 2014, GCN Report, 466

Lipunov, V. M., Krylov, A. V., Kornilov, V. G., et al. 2004, Astron. Nachr., 325, 580

Lipunov, V. M., Gorosabel, J., Pruzhinskaya, M. V., et al. 2016, MNRAS, 455, 712

Lithwick, Y., \& Sari, R. 2001, ApJ, 555, 540

Liu, B., Chen, W., Liang, Y.-F., et al. 2014, ApJ, 787, L6

Lü, J., Zou, Y.-C., Lei, W.-H., et al. 2012, ApJ, 751, 49

Maiorano, E., Masetti, N., Palazzi, E., et al. 2006, A\&A, 455, 423

Margutti, R., Guidorzi, C., Chincarini, G., et al. 2010, MNRAS, 406, 2149

Marshall, F. E., \& Cannizzo, J. K. 2015, GRB Coordinates Network, 17820

Marshall, F. E., vanden Berk, D. E., \& Racusin, J. 2007, GRB Coordinates Network, 6041

Martin-Carrillo, A., Hanlon, L., Topinka, M., et al. 2014, A\&A, 567, A84

Mazaeva, E., Klunko, E., Volnova, A., Korobtsev, I., \& Pozanenko, A. 2015, GRB Coordinates Network, 18175

Melandri, A., Kobayashi, S., Mundell, C. G., et al. 2010, ApJ, 723, 1331

Melandri, A., Covino, S., Rogantini, D., et al. 2014a, A\&A, 565, A72

Melandri, A., Virgili, F. J., Guidorzi, C., et al. 2014b, A\&A, 572, A55

Mesler, R. A., Pihlström, Y. M., Taylor, G. B., \& Granot, J. 2012, ApJ, 759, 4

Mimica, P., Giannios, D., \& Aloy, M. A. 2009, A\&A, 494, 879

Molinari, E., Vergani, S. D., Malesani, D., et al. 2007, A\&A, 469, L13

Nappo, F., Ghisellini, G., Ghirlanda, G., et al. 2014, MNRAS, 445, 1625

Nardini, M., Ghisellini, G., Ghirlanda, G., \& Celotti, A. 2010, MNRAS, 403, 1131

Nardini, M., Elliott, J., Filgas, R., et al. 2014, A\&A, 562, A29

Nava, L., Sironi, L., Ghisellini, G., Celotti, A., \& Ghirlanda, G. 2013, MNRAS, 433, 2107

Nava, L., Vianello, G., Omodei, N., et al. 2014, MNRAS, 443, 3578

Nava, L., Desiante, R., Longo, F., et al. 2017, MNRAS, 465, 811

Oates, S. R., \& Hagen, L. M. Z. 2015, GRB Coordinates Network, 17424

Oates, S. R., \& Racusin, J. L. 2012, GRB Coordinates Network, 13725

Paczynski, B. 1986, ApJ, 308, L43

Page, K. L., Starling, R. L. C., Fitzpatrick, G., et al. 2011, MNRAS, 416, 2078

Panaitescu, A. 2008, MNRAS, 383, 1143

Panaitescu, A. 2017, ApJ, 837, 13

Panaitescu, A., \& Kumar, P. 2000, ApJ, 543, 66

Park, H. S., Ables, E., Band, D. L., et al. 1997, ApJ, 490, 99

Perley, D. A., Bloom, J. S., Butler, N. R., et al. 2008a, ApJ, 672, 449

Perley, D. A., Li, W., Chornock, R., et al. 2008b, ApJ, 688, 470

Pihlström, Y. M., Taylor, G. B., Granot, J., \& Doeleman, S. 2007, ApJ, 664, 411

Piran, T. 1999, Phys. Rep., 314, 575

Price, P. A., Kulkarni, S. R., Berger, E., et al. 2003, ApJ, 589, 838

Racusin, J. L., Karpov, S. V., Sokolowski, M., et al. 2008, Nature, 455, 183

Romano, P., Campana, S., Chincarini, G., et al. 2006, A\&A, 456, 917

Rossi, A., Schulze, S., Klose, S., et al. 2011, A\&A, 529, A142

Rykoff, E. S., Aharonian, F., Akerlof, C. W., et al. 2009, ApJ, 702, 489

Sahu, K. C., Vreeswijk, P., Bakos, G., et al. 2000, ApJ, 540, 74

Sakamoto, T., Barthelmy, S. D., Baumgartner, W. H., et al. 2011, ApJS, 195, 2

Salvaterra, R., Della Valle, M., Campana, S., et al. 2009, Nature, 461, 1258

Salvaterra, R., Campana, S., Vergani, S. D., et al. 2012, ApJ, 749, 68

Sánchez-Ramírez, R., Hancock, P. J., Jóhannesson, G., et al. 2017, MNRAS, 464, 4624

Santana, R., Barniol Duran, R., \& Kumar, P. 2014, ApJ, 785, 29 
Sari, R., \& Piran, T. 1999, ApJ, 520, 641

Sari, R., Piran, T., \& Narayan, R. 1998, ApJ, 497, L17

Sato, R., Kawai, N., Suzuki, M., et al. 2003, ApJ, 599, L9

Siegel, M. H., \& Gompertz, B. P. 2014, GRB Coordinates Network, 16219

Singer, L. P., Cenko, S. B., Kasliwal, M. M., Fremling, C., \& Dzigan, Y. 2014, GRB Coordinates Network, 16226

Soderberg, A. M., Kulkarni, S. R., Price, P. A., et al. 2006, ApJ, 636, 391

Stanek, K. Z., Garnavich, P. M., Kaluzny, J., Pych, W., \& Thompson, I. 1999, ApJ, 522, L39

Stanek, K. Z., Garnavich, P. M., Jha, S., et al. 2001, ApJ, 563, 592

Steele, I. A., Mundell, C. G., Smith, R. J., Kobayashi, S., \& Guidorzi, C. 2009, Nature, 462, 767

Still, M., Roming, P. W. A., Mason, K. O., et al. 2005, ApJ, 635, 1187

Tang, Q.-W., Peng, F.-K., Wang, X.-Y., \& Tam, P.-H. T. 2015, ApJ, 806, 194

Taylor, G. B., Frail, D. A., Berger, E., \& Kulkarni, S. R. 2004, ApJ, 609, L1

Taylor, G. B., Momjian, E., Pihlström, Y., Ghosh, T., \& Salter, C. 2005, ApJ, 622,986

Thöne, C. C., Kann, D. A., Jóhannesson, G., et al. 2010, A\&A, 523, A70

Toma, K., Ioka, K., Yamazaki, R., \& Nakamura, T. 2006, ApJ, 640, L139

Trotter, A., Frank, N., Lacluyze, A., et al. 2013a, GRB Coordinates Network, 14375

Trotter, A., Frank, N., Lacluyze, A., et al. 2013b, GRB Coordinates Network, 14427

Trotter, A., Reichart, D., Haislip, J., et al. 2013c, GRB Coordinates Network, 15491

Trotter, A., Lacluyze, A., Haislip, J., et al. 2014, GRB Coordinates Network, 16304

Trotter, A., Reichart, D., Haislip, J., et al. 2015, GRB Coordinates Network, 18472

Urata, Y., Tashiro, M. S., Tamagawa, T., et al. 2011, PASJ, 63, 137

Veres, P., Corsi, A., Frail, D. A., Cenko, S. B., \& Perley, D. A. 2015, ApJ, 810,
Vestrand, W. T., Borozdin, K. N., Brumby, S. P., et al. 2002, in Advanced Global Communications Technologies for Astronomy II, ed. R. I. Kibrick, Proc. SPIE, 4845, 126

Virgili, F. J., Mundell, C. G., \& Melandri, A. 2013, GRB Coordinates Network, 14785

Volnova, A., Pozanenko, A., Mundrzyjewski, W., \& Tereshenko, V. 2014, GRB Coordinates Network, 16809

Waxman, E. 1997, ApJ, 491, L19

Williams, G. G., Blake, C., \& Hartmann, D. 2002, GRB Coordinates Network, 1492

Wren, J., Vestrand, W. T., Wozniak, P., \& Davis, H. 2012, GRB Coordinates Network, 14013

Wu, Q., Zou, Y.-C., Cao, X., Wang, D.-X., \& Chen, L. 2011, ApJ, 740, L21

Yonetoku, D., Murakami, T., Nakamura, T., et al. 2004, ApJ, 609, 935

Yost, S. A., Alatalo, K., Rykoff, E. S., et al. 2006, ApJ, 636, 959

Yost, S. A., Swan, H. F., Rykoff, E. S., et al. 2007, ApJ, 657, 925

Zaninoni, E., Bernardini, M. G., Margutti, R., Oates, S., \& Chincarini, G. 2013, A\&A, 557, A12

Zerbi, R. M., Chincarini, G., Ghisellini, G., et al. 2001, Astron. Nachr., 322, 275

Zhang, B.-B., van Eerten, H., Burrows, D. N., et al. 2015, ApJ, 806, 15

Zhang, Q., Huang, Y. F., \& Zong, H. S. 2016, ApJ, 823, 156

Zhao, X.-H., Li, Z., \& Bai, J.-M. 2011, ApJ, 726, 89

Zheng, W., \& Filippenko, A. V. 2015, GRB Coordinates Network, 17585

Zheng, W., Flewelling, H., Guver, T., \& Yuan, F. 2013, GRB Coordinates Network, 14208

Zheng, W., Filippenko, A. V., Morgan, A., \& Cenko, S. B. 2014a, GRB Coordinates Network, 16137

Zheng, W., Filippenko, A. V., Morgan, A., \& Cenko, S. B. 2014b, GRB Coordinates Network, 16156

Zou, Y.-C., \& Piran, T. 2010, MNRAS, 402, 1854

Zou, Y.-C., Fan, Y.-Z., \& Piran, T. 2011, ApJ, 726, L2 
G. Ghirlanda et al.: Bulk Lorentz factor of GRBs

\section{Appendix A: Additional table}

Table A.1. Sample of GRBs with measured $t_{\mathrm{p}}(69$ events) from the peak of the light curve (those from the LAT light curve are labelled "L").

\begin{tabular}{|c|c|c|c|c|c|c|}
\hline$\overline{\overline{\mathrm{GRB}}}$ & $\overline{z z}$ & $\begin{array}{l}\log E_{\mathrm{p}} \\
\mathrm{keV}\end{array}$ & $\begin{array}{l}\log E_{\text {iso }} \\
\text { erg }\end{array}$ & $\begin{array}{l}\log L_{\text {iso }} \\
\text { erg s }^{-1}\end{array}$ & $\begin{array}{l}\log t_{\mathrm{p}} \\
\mathrm{s}\end{array}$ & Ref. \\
\hline 971214 & 3.42 & $2.836 \pm 0.084$ & $53.324 \pm 0.049$ & $52.858 \pm 0.08$ & $\leq 4.67$ & Diercks et al. (1998) \\
\hline 980326 & 1.0 & $1.851 \pm 0.220$ & $51.683 \pm 0.077$ & $51.54 \pm 0.125$ & $\leq 4.56$ & Groot et al. (1998) \\
\hline 980703 & 0.97 & $2.698 \pm 0.087$ & $52.839 \pm 0.052$ & $52.32 \pm 0.101$ & $\leq 4.88$ & Holland et al. (2001) \\
\hline 990123(g) & 1.6 & $3.308 \pm 0.034$ & $54.378 \pm 0.051$ & $53.548 \pm 0.151$ & 1.68 & Galama et al. (1999) \\
\hline 990510 & 1.62 & $2.626 \pm 0.043$ & $53.25 \pm 0.063$ & $52.787 \pm 0.076$ & $\leq 4.10$ & Stanek et al. (1999) \\
\hline 990712 & 0.43 & $1.968 \pm 0.070$ & $51.827 \pm 0.083$ & $50.873 \pm 0.111$ & $\leq 4.18$ & Sahu et al. (2000) \\
\hline 991216 & 1.02 & $2.808 \pm 0.087$ & $53.829 \pm 0.052$ & $53.053 \pm 0.144$ & $\leq 4.60$ & Halpern et al. (2000) \\
\hline 000926 & 2.07 & $2.491 \pm 0.028$ & $53.431 \pm 0.093$ & $52.675 \pm 0.119$ & $\leq 4.87$ & Fynbo et al. (2001) \\
\hline 010222 & 1.48 & $2.884 \pm 0.017$ & $53.908 \pm 0.0050$ & $51.896 \pm 0.025$ & $\leq 4.11$ & Stanek et al. (2001) \\
\hline 011211 & 2.14 & $2.267 \pm 0.059$ & $52.822 \pm 0.086$ & $51.501 \pm 0.044$ & $\leq 4.59$ & Holland et al. (2002) \\
\hline 020124 & 3.2 & $2.591 \pm 0.126$ & $53.332 \pm 0.147$ & $52.709 \pm 0.172$ & $\leq 3.76$ & Berger et al. (2002) \\
\hline 020405 & 0.69 & $2.79 \pm 0.12$ & $53.097 \pm 0.045$ & $52.14 \pm 0.025$ & $\leq 4.80$ & Price et al. (2003) \\
\hline 020813 & 1.25 & $2.679 \pm 0.086$ & $53.831 \pm 0.064$ & $52.412 \pm 0.04$ & $\leq 3.78$ & Williams et al. (2002) \\
\hline 021211 & 1.01 & $1.973 \pm 0.088$ & $52.041 \pm 0.051$ & $51.853 \pm 0.06$ & $\leq 2.11$ & Li et al. (2003) \\
\hline 030328 & 1.52 & $2.516 \pm 0.046$ & $53.558 \pm 0.048$ & $52.041 \pm 0.061$ & $\leq 3.69$ & Maiorano et al. (2006) \\
\hline 030329 & 0.17 & $1.898 \pm 0.016$ & $52.22 \pm 0.052$ & $51.281 \pm 0.054$ & $\leq 3.61$ & Sato et al. (2 \\
\hline 040924 & 0.86 & $2.009 \pm 0.149$ & $51.978 \pm 0.046$ & $51.785 \pm 0.078$ & $\leq 2.97$ & Soderberg et al. (2006) \\
\hline 041006 & 0.72 & $2.033 \pm 0.088$ & $52.919 \pm 0.068$ & $51.937 \pm 0.068$ & $\leq 3.37$ & Kinugasa \& Torii (2004) \\
\hline 050318 & 1.44 & $2.061 \pm 0.102$ & $52.301 \pm 0.067$ & $51.708 \pm 0.068$ & $\leq 3.51$ & Still et al. (2005) \\
\hline 050401 & 2.9 & $2.7 \pm 0.101$ & $53.613 \pm 0.085$ & $53.307 \pm 0.021$ & $\leq 1.52$ & Melandri et al. (2014a) \\
\hline 050416A & 0.65 & $1.456 \pm 0.126$ & $50.919 \pm 0.152$ & $50.968 \pm 0.042$ & $\leq 2.22$ & Melandri et a \\
\hline $050502 \mathrm{~A}(\mathrm{~g})$ & 3.79 & $2.698 \pm 0.192$ & $52.602 \pm 0.043$ & $52.415 \pm 0.05$ & 1.76 & Yost et al. (2006) \\
\hline $050525 \mathrm{~A}$ & 0.61 & $2.104 \pm 0.019$ & $52.461 \pm 0.086$ & $51.979 \pm 0.114$ & $\leq 1.80$ & Melandri et al. (2014a) \\
\hline 050603 & 2.82 & $3.125 \pm 0.035$ & $53.777 \pm 0.029$ & $54.328 \pm 0.045$ & $\leq 4.52$ & Grupe et al. \\
\hline $050730(\mathrm{~s})$ & 3.97 & $2.989 \pm 0.087$ & $52.857 \pm 0.043$ & $51.845 \pm 0.043$ & 2.77 & Liang et al. \\
\hline $050820 \mathrm{~A}(\mathrm{~g})$ & 2.61 & $3.122 \pm 0.091$ & $53.989 \pm 0.034$ & $52.959 \pm 0.032$ & 2.59 & Liang et al. (2010) \\
\hline 050904 & 6.29 & $3.502 \pm 0.15$ & $54.093 \pm 0.035$ & $53.041 \pm 0.154$ & $\leq 2.30$ & Zaninoni et al. (2013) \\
\hline 050908 & 3.34 & $2.29 \pm 0.08$ & $52.294 \pm 0.071$ & $51.919 \pm 0.068$ & $\leq 2.48$ & Zaninoni et \\
\hline $050922 \mathrm{C}(\mathrm{g})$ & 2.2 & $2.62 \pm 0.123$ & $52.656 \pm 0.075$ & $53.279 \pm 0.0050$ & 2.12 & Ghisellini \\
\hline 051109A & 2.35 & $2.732 \pm 0.307$ & $52.876 \pm 0.051$ & $52.588 \pm 0.043$ & $\leq 1.60$ & Yost et al. (2007) \\
\hline 060124(g) & 2.3 & $2.803 \pm 0.111$ & $53.633 \pm 0.034$ & $53.152 \pm 0.0040$ & 2.80 & Romano et al. (2006) \\
\hline 060206 & 4.05 & $2.581 \pm 0.112$ & $52.67 \pm 0.066$ & $52.746 \pm 0.07$ & $\leq 2.50$ & Melandri et al. (2014a) \\
\hline $060210(\mathrm{~g})$ & 3.91 & $2.76 \pm 0.14$ & $53.618 \pm 0.06$ & $52.775 \pm 0.058$ & 2.83 & Melandri et al. (2014a) \\
\hline $060418(\mathrm{~g})$ & 1.49 & $2.757 \pm 0.087$ & $53.107 \pm 0.034$ & $52.276 \pm 0.037$ & 2.18 & Molinari et al. (2007) \\
\hline 060526 & 3.21 & $2.022 \pm 0.087$ & $52.412 \pm 0.044$ & $52.236 \pm 0.078$ & $\leq 1.69$ & Thöne et al. (2010) \\
\hline $060605(\mathrm{~g})$ & 3.78 & $2.69 \pm 0.222$ & $52.452 \pm 0.069$ & $51.978 \pm 0.069$ & 2.68 & Rykoff et al. (2009) \\
\hline $060607 \mathrm{~A}(\mathrm{~g})$ & 3.08 & $2.76 \pm 0.151$ & $53.037 \pm 0.062$ & $52.301 \pm 0.059$ & 2.25 & Molinari et al. (2007) \\
\hline 060714 & 2.71 & $2.369 \pm 0.202$ & $53.127 \pm 0.03$ & $52.152 \pm 0.031$ & $\leq 2.31$ & Krimm et al. (2007) \\
\hline 060908 & 2.43 & $2.68 \pm 0.1$ & $52.892 \pm 0.075$ & $52.415 \pm 0.077$ & $\leq 1.84$ & Melandri et al. (2014a) \\
\hline 060927 & 5.6 & $2.675 \pm 0.107$ & $52.98 \pm 0.067$ & $53.057 \pm 0.076$ & $\leq 1.22$ & Melandri et al. (2014a) \\
\hline 061007(g) & 1.26 & $2.955 \pm 0.021$ & $53.945 \pm 0.048$ & $53.241 \pm 0.061$ & 1.87 & Melandri et al. (2014a) \\
\hline 061021 & 0.35 & $2.89 \pm 0.22$ & $51.633 \pm 0.131$ & $51.212 \pm 0.107$ & $\leq 1.91$ & Melandri et al. (2014a) \\
\hline $061121(\mathrm{~g})$ & 1.31 & $3.11 \pm 0.052$ & $53.417 \pm 0.05$ & $53.149 \pm 0.0050$ & 2.21 & Melandri et al. (2014a) \\
\hline 061126 & 1.16 & $3.126 \pm 0.133$ & $52.889 \pm 0.045$ & $52.549 \pm 0.037$ & $\leq 1.51$ & Perley et al. (2008a) \\
\hline $061222 \mathrm{~A}$ & 2.09 & $3.037 \pm 0.067$ & $53.778 \pm 0.043$ & $53.146 \pm 0.117$ & $\leq 3.87$ & Melandri et al. (2014a) \\
\hline $070110(\mathrm{~g})$ & 2.35 & $2.568 \pm 0.2$ & $52.74 \pm 0.118$ & $51.654 \pm 0.072$ & 3.07 & Ghisellini et al. (2009) \\
\hline 070125 & 1.55 & $2.97 \pm 0.069$ & $53.968 \pm 0.043$ & $53.511 \pm 0.067$ & $\leq 4.67$ & Marshall et al. (2007) \\
\hline 070318(s) & 0.84 & $2.556 \pm 0.087$ & $51.681 \pm 0.043$ & $50.643 \pm 0.043$ & 2.48 & Liang et al. (2010) \\
\hline 070411(s) & 2.95 & $2.676 \pm 0.087$ & $52.681 \pm 0.043$ & $51.724 \pm 0.043$ & 2.65 & Liang et al. (2010) \\
\hline 070419A(s) & 0.97 & $1.724 \pm 0.087$ & $51.204 \pm 0.043$ & $49.519 \pm 0.043$ & 2.77 & Liang et al. (2010) \\
\hline 071003 & 1.1 & $3.225 \pm 0.06$ & $53.255 \pm 0.034$ & $52.924 \pm 0.0080$ & $\leq 1.62$ & Perley et al. (2008b) \\
\hline 071010A(s) & 0.99 & $1.869 \pm 0.087$ & $51.114 \pm 0.043$ & $50.415 \pm 0.043$ & 2.62 & Covino et al. (2008) \\
\hline $071010 \mathrm{~B}(\mathrm{~g})$ & 0.95 & $2.004 \pm 0.099$ & $52.326 \pm 0.074$ & $51.806 \pm 0.0040$ & 2.45 & Liang et al. (2013) \\
\hline 071020 & 2.14 & $3.006 \pm 0.088$ & $53.0 \pm 0.061$ & $53.342 \pm 0.019$ & $\leq 1.40$ & Melandri et al. (2014a) \\
\hline $071025(\mathrm{~s})$ & 5.2 & $3.01 \pm 0.087$ & $53.519 \pm 0.043$ & $52.519 \pm 0.043$ & 2.74 & Liang et al. (2013) \\
\hline
\end{tabular}

Notes. $(\mathrm{g})=$ Gold sample, $(\mathrm{s})=$ Silver sample. Upper limits on $t_{\mathrm{p}}$ for $106 \mathrm{GRBs}$ are reported. 
Table A.1. continued.

\begin{tabular}{|c|c|c|c|c|c|c|}
\hline$\overline{\mathrm{GRB}}$ & $z$ & $\begin{array}{l}\log E_{\mathrm{p}} \\
\mathrm{keV}\end{array}$ & $\begin{array}{l}\log E_{\text {iso }} \\
\text { erg }\end{array}$ & $\begin{array}{l}\log L_{\text {iso }} \\
\operatorname{erg~s}^{-1}\end{array}$ & $\begin{array}{l}\log t_{\mathrm{p}} \\
\mathrm{s}\end{array}$ & Ref. \\
\hline 071031(s) & 2.69 & $1.643 \pm 0.087$ & $52.146 \pm 0.043$ & $51.279 \pm 0.043$ & 3.08 & Liang et al. (2013) \\
\hline $071112 \mathrm{C} *(\mathrm{~s})$ & 0.82 & $2.776 \pm 0.087$ & $52.204 \pm 0.043$ & $51.602 \pm 0.043$ & 2.25 & Liang et al. (2013) \\
\hline $080310(\mathrm{~s})$ & 2.42 & $1.875 \pm 0.087$ & $52.491 \pm 0.043$ & $51.591 \pm 0.043$ & 2.26 & Liang et al. (2013) \\
\hline 080319B (g) & 0.94 & $3.101 \pm 0.0090$ & $54.176 \pm 0.049$ & $52.982 \pm 0.01$ & 1.24 & Melandri et al. (2014a) \\
\hline 080319C & 1.95 & $3.244 \pm 0.125$ & $53.176 \pm 0.023$ & $52.978 \pm 0.0050$ & $\leq 1.47$ & Melandri et al. (2014a) \\
\hline 080330(s) & 1.51 & $1.701 \pm 0.087$ & $51.301 \pm 0.043$ & $50.839 \pm 0.043$ & 2.76 & Liang et al. (2013) \\
\hline 080413B & 1.1 & $2.188 \pm 0.094$ & $52.301 \pm 0.076$ & $52.217 \pm 0.079$ & $\leq 1.84$ & Melandri et al. (2014a) \\
\hline 080603B & 2.69 & $2.575 \pm 0.088$ & $53.041 \pm 0.063$ & $53.083 \pm 0.018$ & $\leq 1.36$ & Melandri et al. (2014a) \\
\hline 080605 & 1.64 & $2.823 \pm 0.033$ & $53.403 \pm 0.062$ & $53.507 \pm 0.018$ & $\leq 2.71$ & Melandri et al. (2014a) \\
\hline 080607 & 3.04 & $3.228 \pm 0.044$ & $54.301 \pm 0.028$ & $54.336 \pm 0.2$ & $\leq 1.60$ & Melandri et al. (2014a) \\
\hline 080710(s) & 0.85 & $2.743 \pm 0.087$ & $51.398 \pm 0.043$ & $50.322 \pm 0.043$ & 3.28 & Liang et al. (2013) \\
\hline 080721 & 2.59 & $3.241 \pm 0.056$ & $54.079 \pm 0.043$ & $54.009 \pm 0.064$ & $\leq 2.20$ & Melandri et al. (2014a) \\
\hline 080804(g) & 2.2 & $2.908 \pm 0.024$ & $53.061 \pm 0.076$ & $52.43 \pm 0.052$ & 1.80 & Melandri et al. (2014a) \\
\hline 080810(g) & 3.35 & $3.173 \pm 0.102$ & $53.592 \pm 0.041$ & $52.967 \pm 0.041$ & 2.07 & Liang et al. (2013) \\
\hline 080913 & 6.7 & $2.855 \pm 0.261$ & $52.708 \pm 0.043$ & $52.886 \pm 0.045$ & $\leq 2.78$ & Greiner et al. (2009) \\
\hline 080916A & 0.69 & $2.207 \pm 0.105$ & $52.0 \pm 0.087$ & $50.914 \pm 0.085$ & $\leq 1.62$ & Melandri et al. (2014a) \\
\hline $080916 \mathrm{C}(\mathrm{gL})$ & 4.35 & $3.441 \pm 0.019$ & $54.748 \pm 0.039$ & $54.017 \pm 0.037$ & 0.79 & Ghisellini et al. (2010) \\
\hline 080928(s) & 1.69 & $2.301 \pm 0.087$ & $52.23 \pm 0.043$ & $51.477 \pm 0.043$ & 3.36 & Rossi et al. (2011) \\
\hline 081007(g) & 0.53 & $1.785 \pm 0.107$ & $51.23 \pm 0.026$ & $50.633 \pm 0.04$ & 2.09 & Melandri et al. (2014a) \\
\hline 081008(s) & 1.97 & $2.427 \pm 0.087$ & $52.602 \pm 0.043$ & $51.477 \pm 0.043$ & 2.21 & Liang et al. (2013) \\
\hline 081109A(s) & 0.98 & $2.316 \pm 0.087$ & $52.0 \pm 0.043$ & $50.699 \pm 0.043$ & 2.75 & Liang et al. (2013) \\
\hline 081121 & 2.51 & $2.94 \pm 0.061$ & $53.505 \pm 0.068$ & $53.114 \pm 0.043$ & $\leq 1.80$ & Melandri et al. (2014a) \\
\hline $081203 \mathrm{~A}(\mathrm{~g})$ & 2.1 & $3.188 \pm 0.213$ & $53.544 \pm 0.037$ & $52.449 \pm 0.03$ & 2.49 & Melandri et al. \\
\hline 081222 & 2.77 & $2.694 \pm 0.102$ & $53.398 \pm 0.045$ & $52.903 \pm 0.067$ & $\leq 1.7$ & Melandri et al. (2014a) \\
\hline 090102 & 1.55 & $3.06 \pm 0.054$ & $53.342 \pm 0.051$ & $52.94 \pm 0.028$ & $\leq 1.20$ & Melandri et al. (2014a) \\
\hline 090313(s) & 3.38 & $2.382 \pm 0.087$ & $52.663 \pm 0.043$ & $51.279 \pm 0.043$ & 3.03 & Melandri et al. (2010) \\
\hline 090323(gL) & 3.57 & $3.279 \pm 0.078$ & $54.591 \pm 0.045$ & $53.585 \pm 0.167$ & 2.30 & Ghisellini et al. (2010) \\
\hline 090328(L) & 0.74 & $3.012 \pm 0.132$ & $52.491 \pm 0.042$ & $52.053 \pm 0.012$ & $\leq 1.05$ & Panaitescu (2017) \\
\hline 090418A & 1.61 & $3.201 \pm 0.165$ & $53.17 \pm 0.044$ & $52.033 \pm 0.069$ & $\leq 2.21$ & Henden et al. (2009) \\
\hline 090423 & 8.1 & $2.873 \pm 0.08$ & $53.274 \pm 0.042$ & $53.09 \pm 0.029$ & $\leq 2.38$ & Salvaterra et al. (2009) \\
\hline 090424 & 0.54 & $2.225 \pm 0.081$ & $52.407 \pm 0.051$ & $52.037 \pm 0.0070$ & $\leq 1.94$ & Jin et al. (2013) \\
\hline $090510(\mathrm{gSL})$ & 0.9 & $3.643 \pm 0.039$ & $52.699 \pm 0.043$ & $53.25 \pm 0.029$ & -0.09 & Ghirlanda et al. (2010) \\
\hline 090516 & 4.11 & $2.969 \pm 0.337$ & $53.748 \pm 0.047$ & $52.857 \pm 0.016$ & $\leq 3.01$ & Guidorzi et al. (2009) \\
\hline 090618(g) & 0.54 & $2.192 \pm 0.031$ & $53.403 \pm 0.043$ & $52.312 \pm 0.018$ & 1.96 & Page et al. (2011) \\
\hline 090709A & 1.8 & $2.474 \pm 0.039$ & $53.86 \pm 0.011$ & $52.943 \pm 0.066$ & $\leq 2.10$ & Melandri et al. (2014a) \\
\hline 090715B & 3.0 & $2.729 \pm 0.133$ & $53.328 \pm 0.041$ & $52.915 \pm 0.121$ & $\leq 2.80$ & Melandri et al. (2014a) \\
\hline 090812(g) & 2.45 & $3.306 \pm 0.142$ & $53.605 \pm 0.043$ & $52.98 \pm 0.044$ & 1.68 & Melandri et al. (2014a) \\
\hline 090902B(gL) & 1.82 & $3.305 \pm 0.0040$ & $54.643 \pm 0.0030$ & $53.77 \pm 0.0070$ & 0.93 & Ghisellini et al. (2010) \\
\hline 090926A $(\mathrm{gL})$ & 2.11 & $2.958 \pm 0.0030$ & $54.301 \pm 0.011$ & $53.869 \pm 0.0090$ & 0.91 & Ghisellini et al. (2010) \\
\hline 091003(L) & 0.9 & $2.89 \pm 0.018$ & $52.778 \pm 0.0090$ & $52.378 \pm 0.011$ & $\leq 0.6$ & Ghisellini et al. (2010) \\
\hline 091018 & 0.97 & $1.74 \pm 0.205$ & $51.901 \pm 0.051$ & $51.673 \pm 0.096$ & $\leq 2.14$ & Melandri et al. (2014a) \\
\hline 091020(g) & 1.71 & $2.705 \pm 0.058$ & $52.898 \pm 0.064$ & $52.519 \pm 0.066$ & 2.13 & Melandri et al. (2014a) \\
\hline 091029(g) & 2.75 & $2.362 \pm 0.125$ & $52.869 \pm 0.043$ & $52.121 \pm 0.024$ & 2.61 & Filgas et al. (2012) \\
\hline 091127 & 0.49 & $1.708 \pm 0.013$ & $52.207 \pm 0.0$ & $51.957 \pm 0.011$ & $\leq 1.87$ & Melandri et al. (2014a) \\
\hline 091208B(L) & 1.06 & $2.389 \pm 0.027$ & $52.292 \pm 0.013$ & $52.238 \pm 0.016$ & $\leq 1.92$ & Melandri et al. (2014a) \\
\hline $100414 \mathrm{~A}(\mathrm{gL})$ & 1.39 & $3.172 \pm 0.0080$ & $53.886 \pm 0.039$ & $52.845 \pm 0.043$ & 1.54 & Panaitescu (2017) \\
\hline $100621 \mathrm{~A}$ & 0.54 & $2.166 \pm 0.068$ & $52.64 \pm 0.05$ & $51.5 \pm 0.033$ & $\leq 2.65$ & Melandri et al. (2014a) \\
\hline $100728 \mathrm{~A}(\mathrm{~L})$ & 1.57 & $2.958 \pm 0.0080$ & $53.929 \pm 0.041$ & $52.568 \pm 0.012$ & $\leq 2.599$ & Ackermann et al. (2013) \\
\hline $100728 \mathrm{~B}(\mathrm{~g})$ & 2.11 & $2.606 \pm 0.031$ & $52.477 \pm 0.043$ & $52.27 \pm 0.028$ & 1.53 & Melandri et al. (2014a) \\
\hline $100814 \mathrm{~A}$ & 1.44 & $2.537 \pm 0.019$ & $52.914 \pm 0.042$ & $51.964 \pm 0.028$ & 2.77 & Nardini et al. (2014) \\
\hline $100901 \mathrm{~A} *(\mathrm{~b})$ & 1.41 & $2.501 \pm 0.087$ & $52.342 \pm 0.043$ & $50.973 \pm 0.043$ & 3.1 & Liang et al. (2013) \\
\hline $100906 \mathrm{~A}(\mathrm{~g})$ & 1.73 & $2.199 \pm 0.044$ & $53.524 \pm 0.039$ & $52.389 \pm 0.015$ & 2.0 & Liang et al. (2013) \\
\hline $110205 \mathrm{~A}(\mathrm{~g})$ & 2.22 & $2.854 \pm 0.145$ & $53.748 \pm 0.047$ & $52.398 \pm 0.06$ & 2.91 & Melandri et al. (2014a) \\
\hline $110213 \mathrm{~A}(\mathrm{~s})$ & 1.46 & $2.382 \pm 0.023$ & $52.806 \pm 0.041$ & $52.32 \pm 0.012$ & 2.51 & Cucchiara et al. (2011) \\
\hline $110422 \mathrm{~A}$ & 1.77 & $2.624 \pm 0.014$ & $53.863 \pm 0.042$ & $53.446 \pm 0.054$ & $\leq 1.77$ & Gorbovskoy et al. 2011 \\
\hline $110503 \mathrm{~A}$ & 1.61 & $2.757 \pm 0.039$ & $53.255 \pm 0.048$ & $53.255 \pm 0.043$ & $\leq 2.44$ & Melandri et al. (2014a) \\
\hline $110715 \mathrm{~A}$ & 0.82 & $2.339 \pm 0.044$ & $52.681 \pm 0.045$ & $52.622 \pm 0.045$ & $\leq 1.93$ & Sánchez-Ramírez et al. (2017) \\
\hline $110731(\mathrm{gL})$ & 2.83 & $3.084 \pm 0.014$ & $53.602 \pm 0.043$ & $53.431 \pm 0.048$ & 0.7 & Ackermann et al. (2013) \\
\hline $111107 \mathrm{~A}$ & 2.89 & $2.623 \pm 0.129$ & $52.477 \pm 0.072$ & $52.236 \pm 0.077$ & $\leq 2.94$ & Lacluyze et al. (2011) \\
\hline
\end{tabular}


G. Ghirlanda et al.: Bulk Lorentz factor of GRBs

Table A.1. continued.

\begin{tabular}{|c|c|c|c|c|c|c|}
\hline$\overline{\overline{\text { GRB }}}$ & $z$ & $\begin{array}{l}\log E_{\mathrm{p}} \\
\mathrm{keV}\end{array}$ & $\begin{array}{l}\log E_{\text {iso }} \\
\text { erg }\end{array}$ & $\begin{array}{l}\log L_{\text {iso }} \\
\mathrm{erg} \mathrm{s}^{-1}\end{array}$ & $\begin{array}{l}\log t_{\mathrm{p}} \\
\mathrm{S}\end{array}$ & Ref. \\
\hline$\overline{111228 \mathrm{~A}}$ & 0.71 & $1.766 \pm 0.051$ & $52.613 \pm 0.048$ & $51.766 \pm 0.05$ & $\leq 1.81$ & Melandri priv. com \\
\hline 120119A & 1.73 & $2.62 \pm 0.057$ & $53.531 \pm 0.064$ & $52.9 \pm 0.063$ & $\leq 1.51$ & Melandri priv. com \\
\hline $120326 \mathrm{~A}$ & 1.8 & $2.061 \pm 0.072$ & $52.556 \pm 0.072$ & $52.017 \pm 0.106$ & $\leq 2.12$ & Melandri et al. (2014b) \\
\hline $120711 \mathrm{~A}(\mathrm{~g})$ & 1.4 & $3.369 \pm 0.016$ & $54.176 \pm 0.043$ & $53.182 \pm 0.015$ & 2.38 & Martin-Carrillo et al. (2014) \\
\hline $120811 \mathrm{C}$ & 2.67 & $2.297 \pm 0.024$ & $52.732 \pm 0.018$ & $52.35 \pm 0.048$ & $\leq 3.01$ & Denisenko et al. (2012) \\
\hline $120815 \mathrm{~A}(\mathrm{~s})$ & 2.36 & $2.19 \pm 0.087$ & $52.086 \pm 0.043$ & $51.954 \pm 0.043$ & 2.64 & Krühler et al. (2013) \\
\hline 120907A & 0.97 & $2.484 \pm 0.093$ & $51.301 \pm 0.065$ & $51.38 \pm 0.09$ & $\leq 2.34$ & Oates \& Racusin (2012) \\
\hline $120909 \mathrm{~A}(\mathrm{~g})$ & 3.93 & $3.261 \pm 0.164$ & $53.863 \pm 0.042$ & $52.857 \pm 0.084$ & 2.46 & Melandri priv. comm. \\
\hline $120922 \mathrm{~A}(\mathrm{~g})$ & 3.1 & $2.193 \pm 0.045$ & $53.301 \pm 0.065$ & $52.462 \pm 0.06$ & 2.95 & Kuin \& Yershov (2012) \\
\hline $121128 \mathrm{~A}(\mathrm{~g})$ & 2.2 & $2.297 \pm 0.033$ & $53.146 \pm 0.031$ & $52.806 \pm 0.034$ & 1.87 & Wren et al. (2012) \\
\hline $121211 \mathrm{~A}$ & 1.02 & $2.288 \pm 0.058$ & $51.114 \pm 0.043$ & $51.079 \pm 0.054$ & $\leq 2.35$ & Japelj et al. (2012) \\
\hline $130215 \mathrm{~A}(\mathrm{~g})$ & 0.6 & $2.394 \pm 0.177$ & $52.398 \pm 0.035$ & $50.924 \pm 0.041$ & 2.87 & Zheng et al. (2013) \\
\hline $130408 \mathrm{~A}$ & 3.76 & $3.002 \pm 0.06$ & $53.447 \pm 0.082$ & $53.763 \pm 0.052$ & $\leq 3.75$ & Trotter et al. (2013a) \\
\hline $130420 \mathrm{~A}(\mathrm{~g})$ & 1.3 & $2.111 \pm 0.024$ & $52.857 \pm 0.042$ & $51.544 \pm 0.037$ & 2.551 & Trotter et al. (2013b) \\
\hline $130427 \mathrm{~A}(\mathrm{gL})$ & 0.34 & $3.139 \pm 0.0030$ & $53.908 \pm 0.043$ & $53.431 \pm 0.048$ & 1.34 & Ackermann et al. (2014) \\
\hline $130505 \mathrm{~A}$ & 2.27 & $3.314 \pm 0.021$ & $54.748 \pm 0.0090$ & $54.602 \pm 0.018$ & $\leq 2.26$ & de Pasquale \& Cannizzo (2013) \\
\hline 130606A & 5.91 & $3.308 \pm 0.103$ & $53.431 \pm 0.048$ & $53.362 \pm 0.038$ & $\leq 3.43$ & Virgili et al. (2013) \\
\hline $130610 \mathrm{~A}(\mathrm{~g})$ & 2.09 & $2.96 \pm 0.063$ & $52.833 \pm 0.045$ & $52.38 \pm 0.09$ & 2.31 & Trotter et al. (2013b) \\
\hline $130612 \mathrm{~A}(\mathrm{~g})$ & 2.01 & $2.27 \pm 0.075$ & $51.855 \pm 0.067$ & $51.942 \pm 0.045$ & 2.04 & Trotter et al. (2013c) \\
\hline $130701 \mathrm{~A}$ & 1.15 & $2.283 \pm 0.02$ & $52.431 \pm 0.021$ & $52.602 \pm 0.033$ & $\leq 2.44$ & Breeveld \& Kuin (2013) \\
\hline $130831 \mathrm{~A}(\mathrm{~g})$ & 0.48 & $1.908 \pm 0.032$ & $51.879 \pm 0.029$ & $51.471 \pm 0.054$ & 2.86 & Zhang et al. (2016) \\
\hline $130907 \mathrm{~A}$ & 1.24 & $2.945 \pm 0.012$ & $54.502 \pm 0.03$ & $53.272 \pm 0.019$ & $\leq 1.64$ & Veres et al. (2015) \\
\hline $131030 \mathrm{~A}$ & 1.29 & $2.593 \pm 0.025$ & $53.477 \pm 0.029$ & $53.0 \pm 0.043$ & $\leq 2.83$ & King et al. (2014) \\
\hline $131108 \mathrm{~A}$ & 2.4 & $3.103 \pm 0.016$ & $53.778 \pm 0.0070$ & $53.301 \pm 0.022$ & $\leq 4.39$ & Gorosabel et al. (2013) \\
\hline $131117 \mathrm{~A}$ & 4.04 & $2.346 \pm 0.072$ & $52.0 \pm 0.087$ & $51.919 \pm 0.068$ & $\leq 1.95$ & Trotter et al. (2013c) \\
\hline $131231 \mathrm{~A}(\mathrm{~g})$ & 0.64 & $2.38 \pm 0.0090$ & $51.544 \pm 0.0010$ & $52.23 \pm 0.0080$ & 2.0 & Liu et al. (2014) \\
\hline $140206 \mathrm{~A}$ & 2.73 & $3.044 \pm 0.011$ & $54.336 \pm 0.0040$ & $53.771 \pm 0.01$ & $\leq 2.477$ & Lien et al. (2014) \\
\hline $140213 \mathrm{~A}$ & 1.21 & $2.344 \pm 0.018$ & $52.908 \pm 0.027$ & $52.663 \pm 0.047$ & $\leq 2.04$ & Trotter et al. (2014d) \\
\hline $140226 \mathrm{~A}$ & 1.98 & $3.091 \pm 0.083$ & $52.748 \pm 0.085$ & $52.405 \pm 0.091$ & $\leq 2.94$ & Cenko et al. (2015) \\
\hline $140301 \mathrm{~A}(\mathrm{~g})$ & 1.42 & $2.093 \pm 0.035$ & $51.623 \pm 0.041$ & $51.0 \pm 0.043$ & $\leq 4.25$ & Kruehler et al. (2014) \\
\hline $140304 \mathrm{~A}$ & 5.28 & $3.067 \pm 0.082$ & $53.124 \pm 0.033$ & $52.944 \pm 0.074$ & $\leq 1.91$ & Gorbovskoy et al. (2014c) \\
\hline 140419A & 3.96 & $3.162 \pm 0.124$ & $54.158 \pm 0.151$ & $53.756 \pm 0.152$ & $\leq 2.28$ & Zheng et al. (2014a) \\
\hline $140423 \mathrm{~A}(\mathrm{~g})$ & 3.26 & $2.727 \pm 0.031$ & $53.748 \pm 0.023$ & $52.753 \pm 0.049$ & 2.30 & Zheng et al. (2014b) \\
\hline $140506 \mathrm{~A}$ & 0.89 & $2.097 \pm 0.236$ & $52.041 \pm 0.039$ & $52.0 \pm 0.087$ & $\leq 2.26$ & Siegel \& Gompertz (2014) \\
\hline $140508 \mathrm{~A}$ & 1.02 & $2.715 \pm 0.02$ & $53.358 \pm 0.013$ & $52.851 \pm 0.024$ & $\leq 4.38$ & Singer et al. (2014) \\
\hline $140512 \mathrm{~A}$ & 0.73 & $3.006 \pm 0.062$ & $52.889 \pm 0.022$ & $51.729 \pm 0.041$ & $\leq 2.24$ & Gorbovskoy et al. (2014b) \\
\hline $140518 \mathrm{~A}$ & 4.71 & $2.4 \pm 0.074$ & $52.74 \pm 0.103$ & $52.279 \pm 0.091$ & $\leq 2.16$ & Trotter et al. (2014) \\
\hline $140620 \mathrm{~A}$ & 2.04 & $2.371 \pm 0.028$ & $52.787 \pm 0.021$ & $52.176 \pm 0.029$ & $\leq 2.95$ & Kasliwal et al. (2014) \\
\hline $140623 \mathrm{~A}$ & 1.92 & $3.009 \pm 0.186$ & $52.875 \pm 0.052$ & $51.851 \pm 0.092$ & $\leq 2.99$ & Bhalerao et al. (2014) \\
\hline $140629 \mathrm{~A}(\mathrm{~g})$ & 2.28 & $2.45 \pm 0.086$ & $52.778 \pm 0.043$ & $52.431 \pm 0.097$ & 2.18 & Gorbovskoy et al. (2014a) \\
\hline 140801A & 1.32 & $2.4 \pm 0.021$ & $52.699 \pm 0.017$ & $52.724 \pm 0.049$ & $\leq 2.03$ & Lipunov et al. (2016) \\
\hline $140808 \mathrm{~A}$ & 3.29 & $2.693 \pm 0.03$ & $52.886 \pm 0.045$ & $53.0 \pm 0.043$ & $\leq 4.07$ & Singer et al. (2014) \\
\hline 140907A & 1.21 & $2.398 \pm 0.026$ & $52.352 \pm 0.015$ & $51.519 \pm 0.039$ & $\leq 3.74$ & Volnova et al. (2014) \\
\hline $141028 \mathrm{~A}$ & 2.33 & $2.921 \pm 0.022$ & $53.778 \pm 0.043$ & $53.27 \pm 0.047$ & $\leq 4.57$ & Burgess et al. (2016) \\
\hline $141109 \mathrm{~A}(\mathrm{~g})$ & 2.93 & $2.875 \pm 0.136$ & $53.491 \pm 0.042$ & $52.623 \pm 0.052$ & 2.98 & Klotz et al. 2014 \\
\hline $141220 \mathrm{~A}$ & 1.32 & $2.433 \pm 0.167$ & $52.255 \pm 0.048$ & $52.072 \pm 0.074$ & $\leq 2.68$ & Gorosabel et al. (2014) \\
\hline $141221 \mathrm{~A}(\mathrm{~g})$ & 1.45 & $2.571 \pm 0.083$ & $52.279 \pm 0.046$ & $51.845 \pm 0.056$ & 2.04 & Bardho et al. (2016) \\
\hline $141225 \mathrm{~A}$ & 0.92 & $2.554 \pm 0.068$ & $52.326 \pm 0.041$ & $51.477 \pm 0.072$ & $\leq 2.32$ & Buckley et al. (2014) \\
\hline $150206 \mathrm{~A}$ & 2.09 & $2.848 \pm 0.068$ & $53.699 \pm 0.043$ & $53.352 \pm 0.058$ & $\leq 2.72$ & Oates \& Hagen (2015) \\
\hline $150301 \mathrm{~B}$ & 1.52 & $2.809 \pm 0.055$ & $52.45 \pm 0.046$ & $51.881 \pm 0.046$ & $\leq 1.90$ & Gorbovskoy et al. (2016) \\
\hline $150314 \mathrm{~A}$ & 1.76 & $2.933 \pm 0.0070$ & $54.255 \pm 0.048$ & $53.914 \pm 0.011$ & $\leq 2.13$ & Zheng \& Filippenko (2015) \\
\hline $150323 \mathrm{~A}$ & 0.59 & $2.179 \pm 0.069$ & $52.114 \pm 0.067$ & $50.352 \pm 0.097$ & $\leq 2.68$ & Cenko \& Perley (2015) \\
\hline $150514 \mathrm{~A}$ & 0.81 & $2.121 \pm 0.036$ & $52.041 \pm 0.039$ & $51.778 \pm 0.029$ & $\leq 4.62$ & Marshall \& Cannizzo (2015) \\
\hline $150818 \mathrm{~A}$ & 0.28 & $2.107 \pm 0.102$ & $51.079 \pm 0.036$ & $49.724 \pm 0.09$ & $\leq 4.14$ & Mazaeva et al. (2015) \\
\hline $150821 \mathrm{~A}$ & 0.75 & $2.788 \pm 0.203$ & $53.164 \pm 0.089$ & $53.0 \pm 0.03$ & $\leq 2.50$ & Kuin et al. (2015) \\
\hline $151021 \mathrm{~A}$ & 2.33 & $2.753 \pm 0.051$ & $54.0 \pm 0.043$ & $53.322 \pm 0.124$ & $\leq 2.15$ & Trotter et al. (2015) \\
\hline $160509 \mathrm{~A}$ & 1.17 & $2.796 \pm 0.069$ & $53.959 \pm 0.043$ & $53.301 \pm 0.033$ & $\leq 4.33$ & Laskar et al. (2016) \\
\hline $160629 \mathrm{~A}(\mathrm{~g})$ & 3.33 & $3.108 \pm 0.028$ & $53.672 \pm 0.028$ & $52.959 \pm 0.076$ & 1.91 & Klotz et al. (2016) \\
\hline
\end{tabular}

